\title{
Modified Epoxy Matrix Resins for Reduced Dependence on Redundant Fasteners in Secondary-Bonded Composite Structures
}

Frank Palmieri' ${ }^{1}$, Tyler Hudson ${ }^{1}$, Austin Smith ${ }^{1}$, Yi Lin², Jin Ho Kang ${ }^{2}$, Isaac Barnett ${ }^{3}$, Bryson Clifford $^{3}$, Roberto Cano ${ }^{1} \&$ John Connell ${ }^{1}$

1. NASA Langley Research Center, Hampton VA

2. National Institute of Aerospace, Hampton, VA

3. NASA Internships Fellowships and Scholarships, Hampton, VA

ACS Spring 2020 National Meeting \& Expo

Philadelphia, PA

March 23'd 2020 


\section{The Challenge}

Global Growth in Aviation

2017

4 BILLION

PASSENGER TRIPS

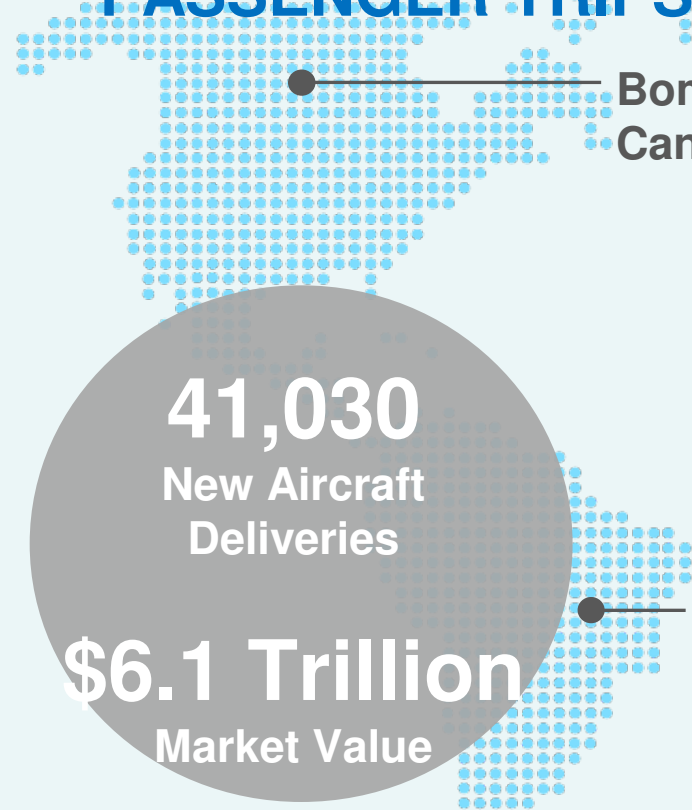

Embraer /

Brazil

bardie

Global Competitors

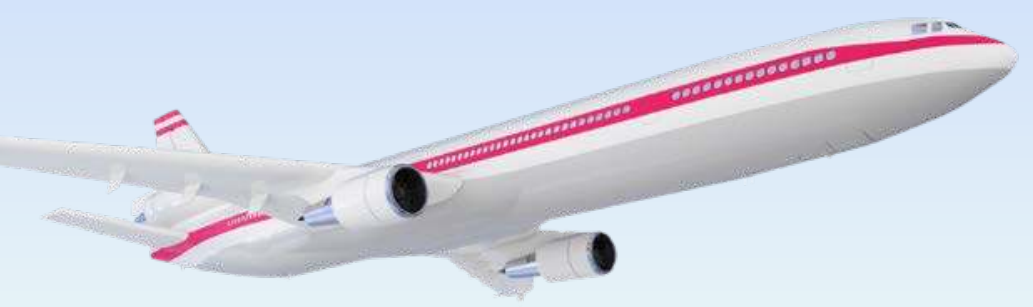

2036

7.8 BILLION

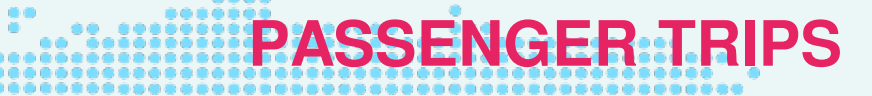

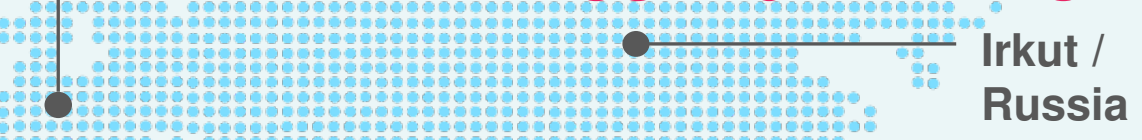
Airbus /

Europe

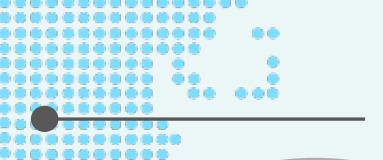

Comac / China

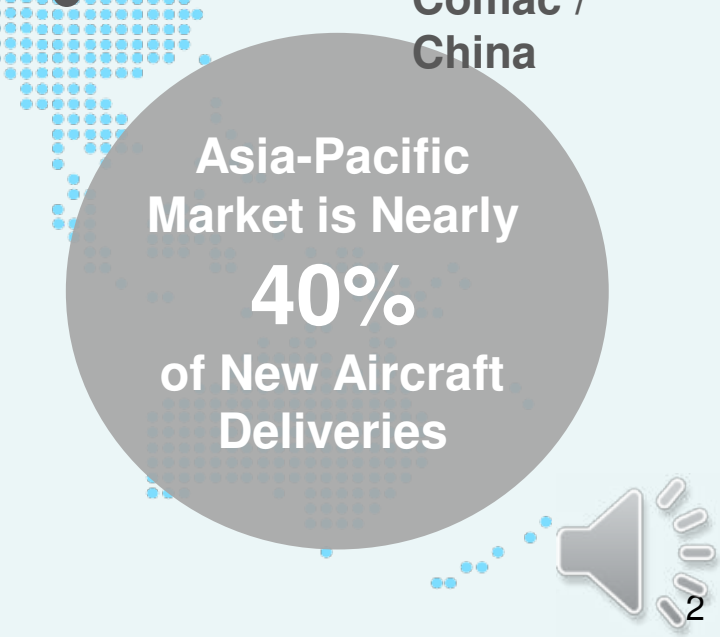




\section{Efficient Composite Airframes}

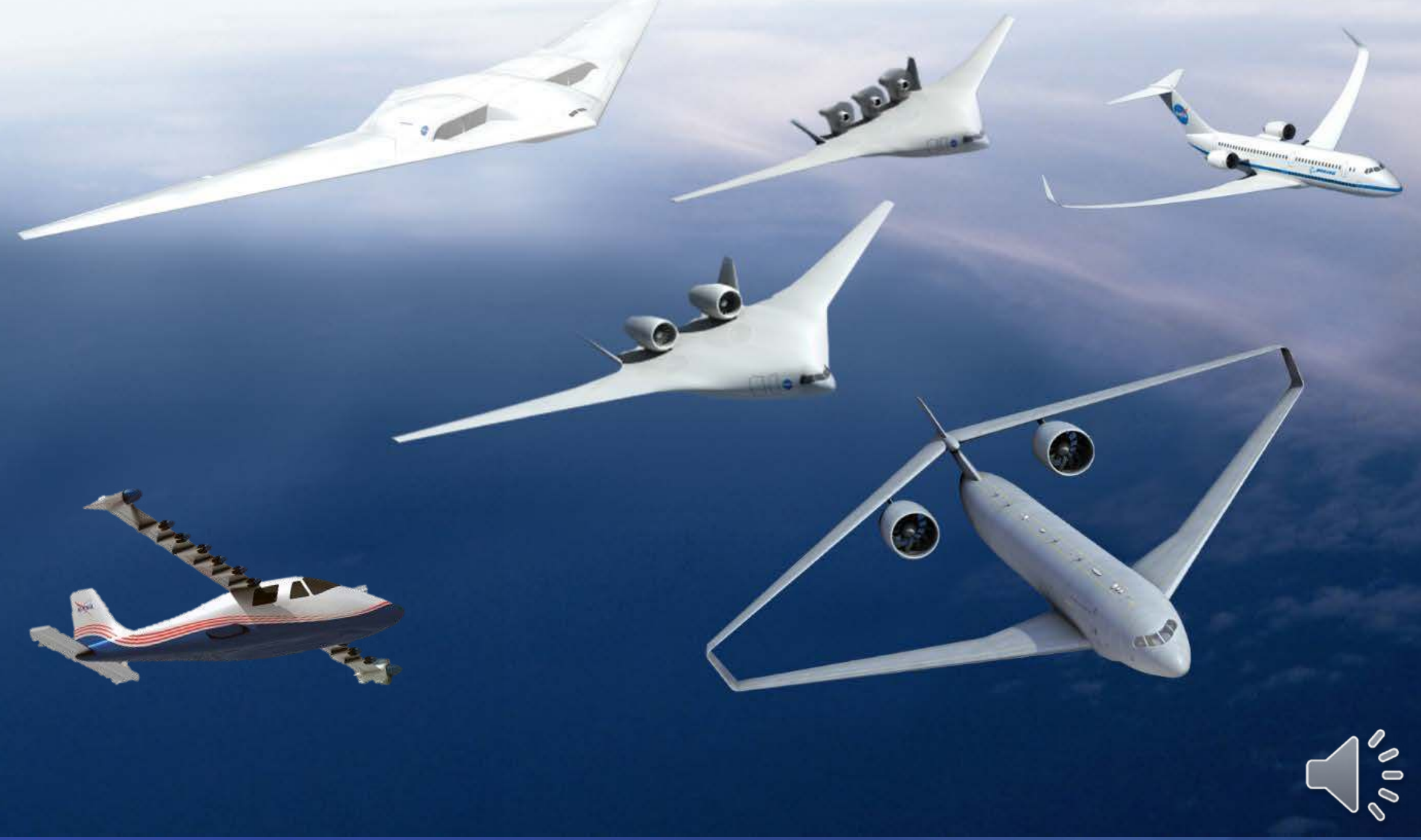




\section{Assembly of Composites}
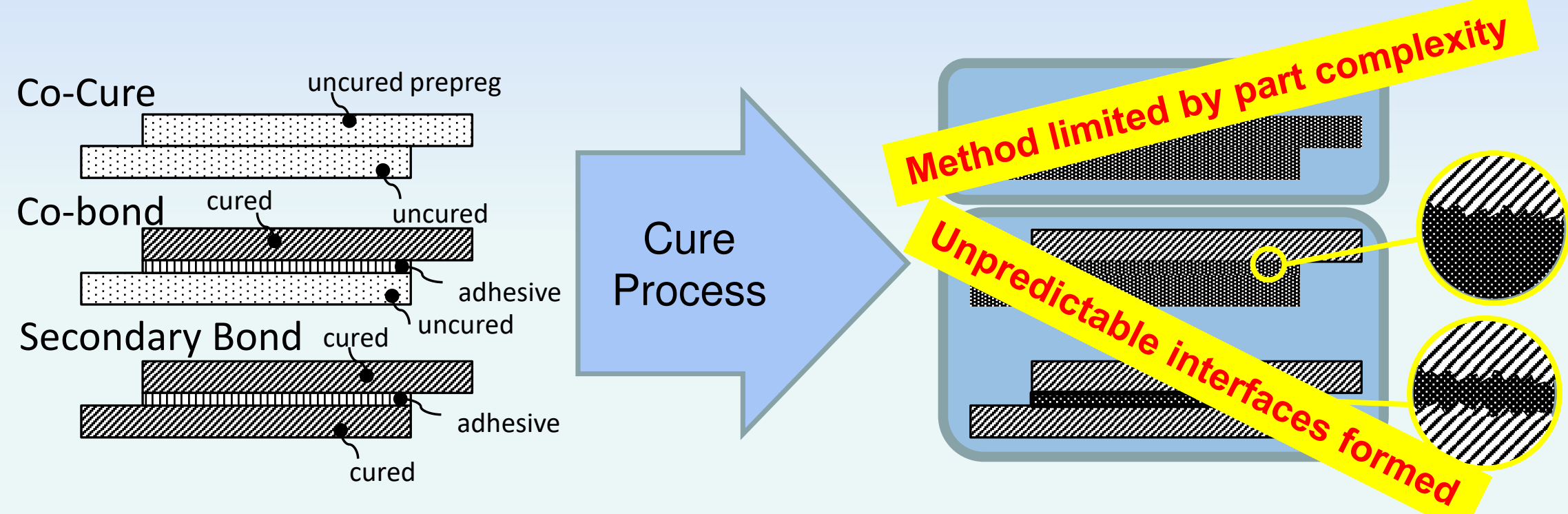

- Co-cure produces predictable components and joints but is limited by complexity

- Bonded/Co-bonded joints are susceptible to weak bonds

- Unpredictable bonds are a concern in primary structure

- A redundant load path is required to ensure structural integrity 


\section{Manufacturing Bottleneck}

Airframes are assemblies of many parts

- Composites can be assembled rapidly with adhesives

- Redundant load path (bolts) is required for certification

- Thousands of drilling and installation steps

Composites should be replacing metals in aircraft but...

- Fastener installation is too slow causing a bottleneck

- Production rates can't meet demand
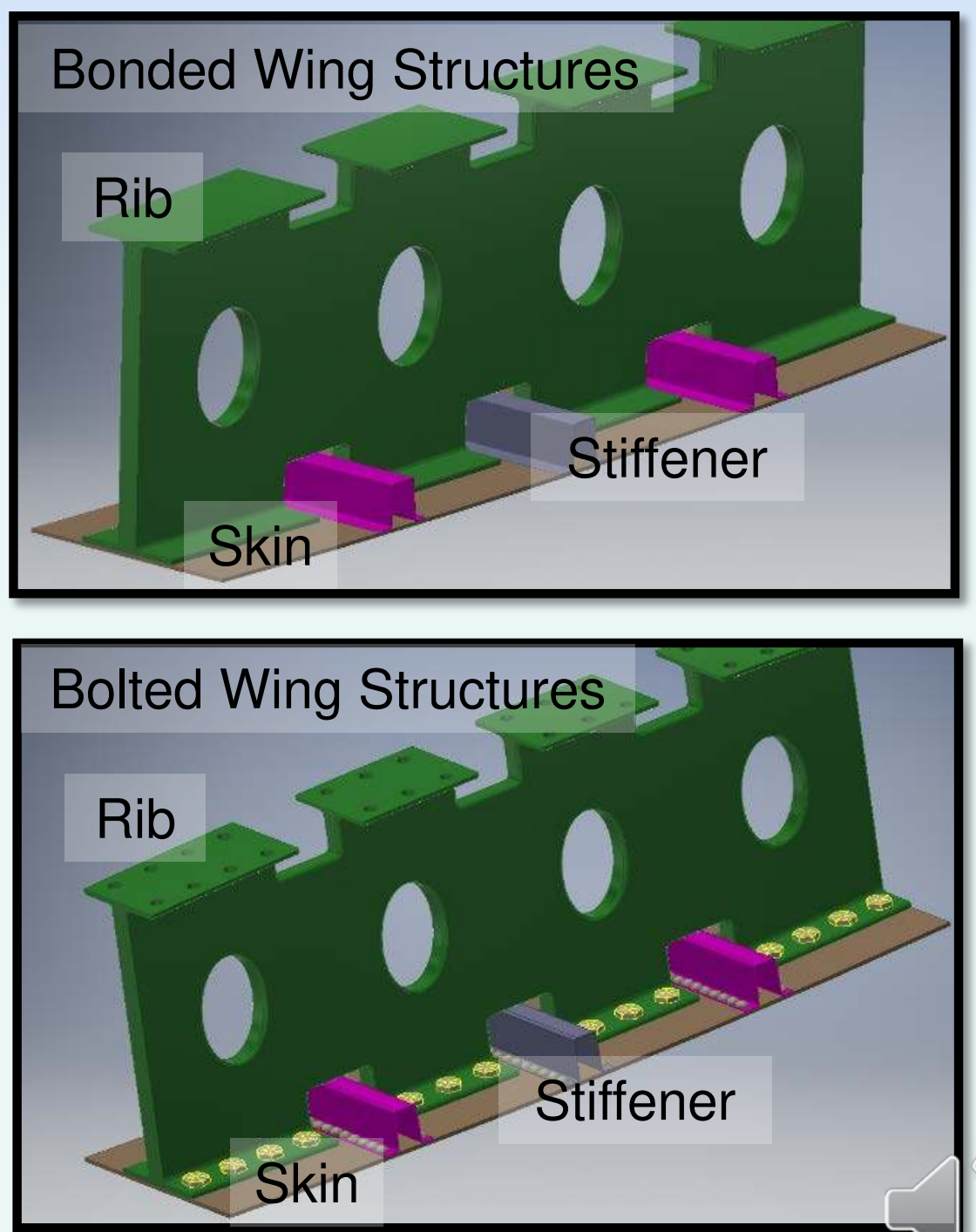


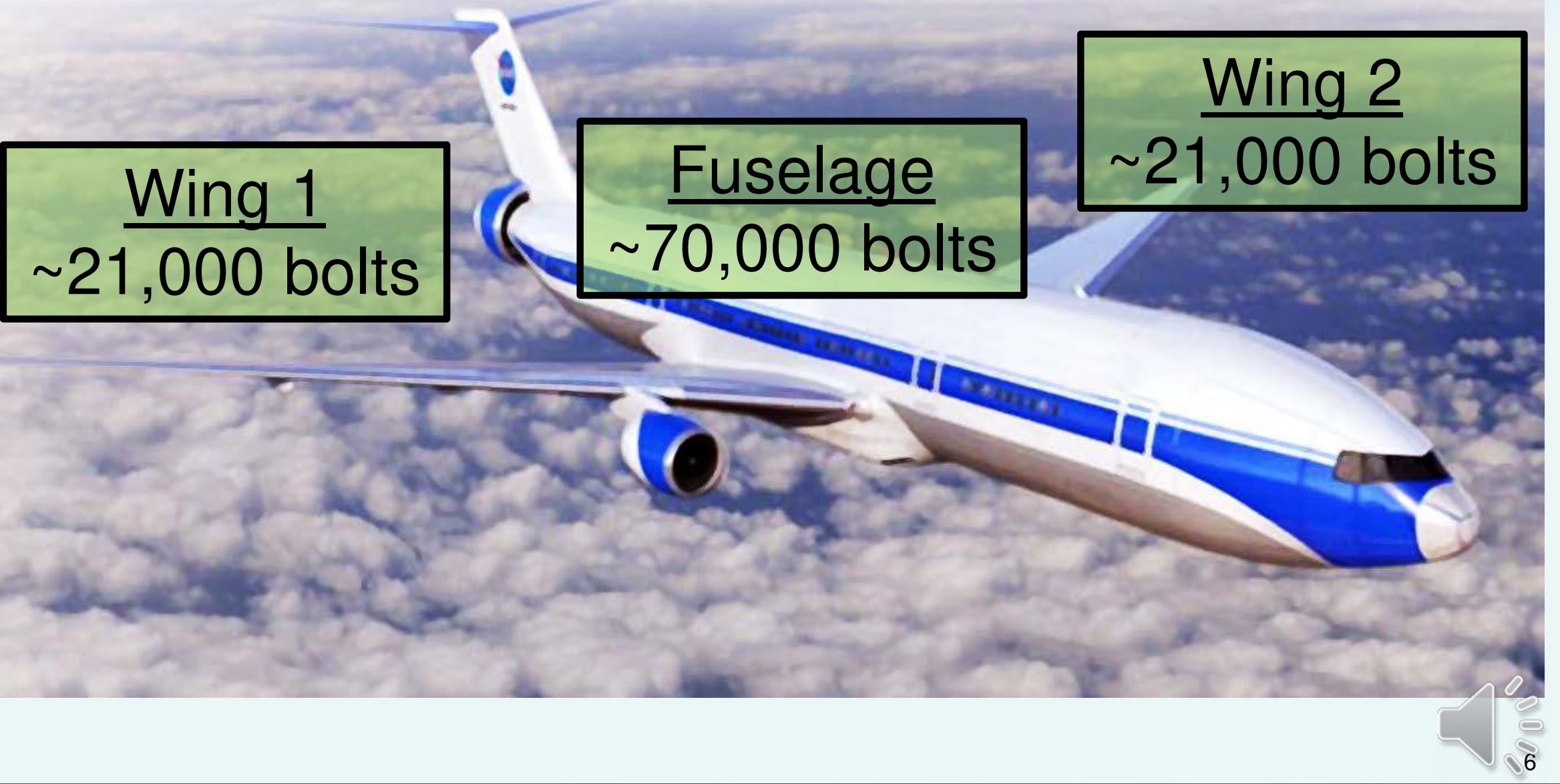




\section{The reliability of co-cure} in a "bonded" assembly

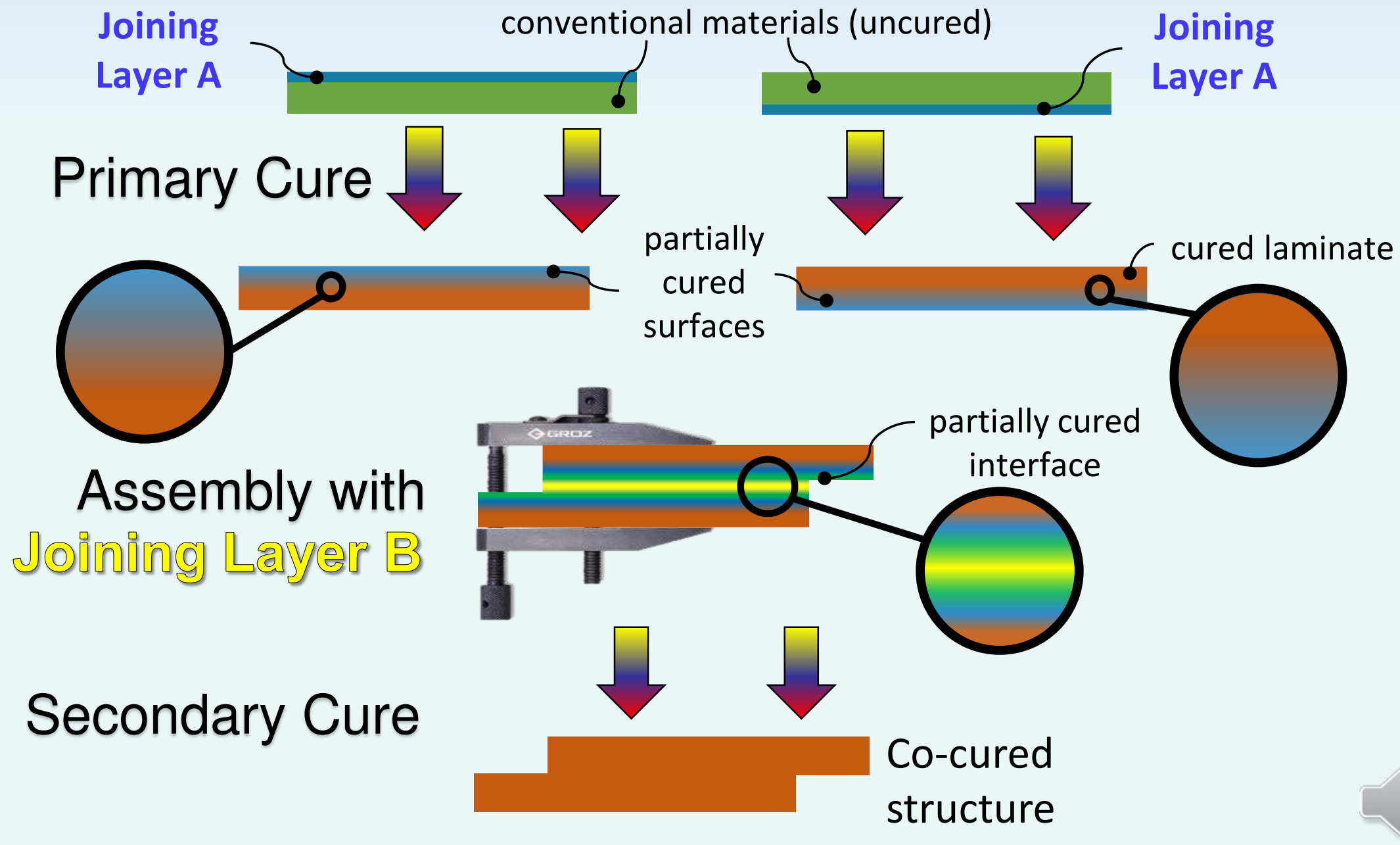




\section{Adses Adhesive Bondline vs. AERoBOND}

Adhesive Bondline

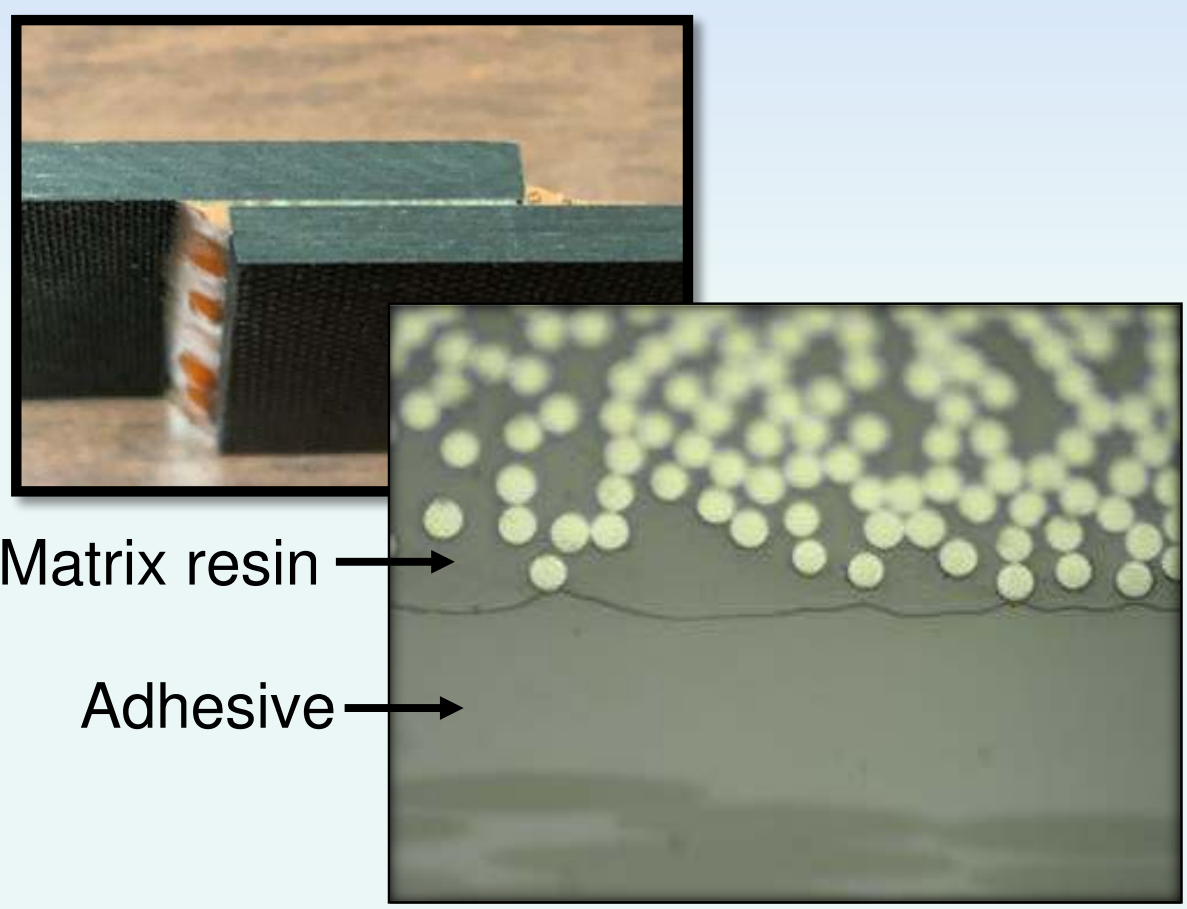

- Cured matrix resin cannot mix with adhesive

Potential for weak bonds
AERoBOND Joint

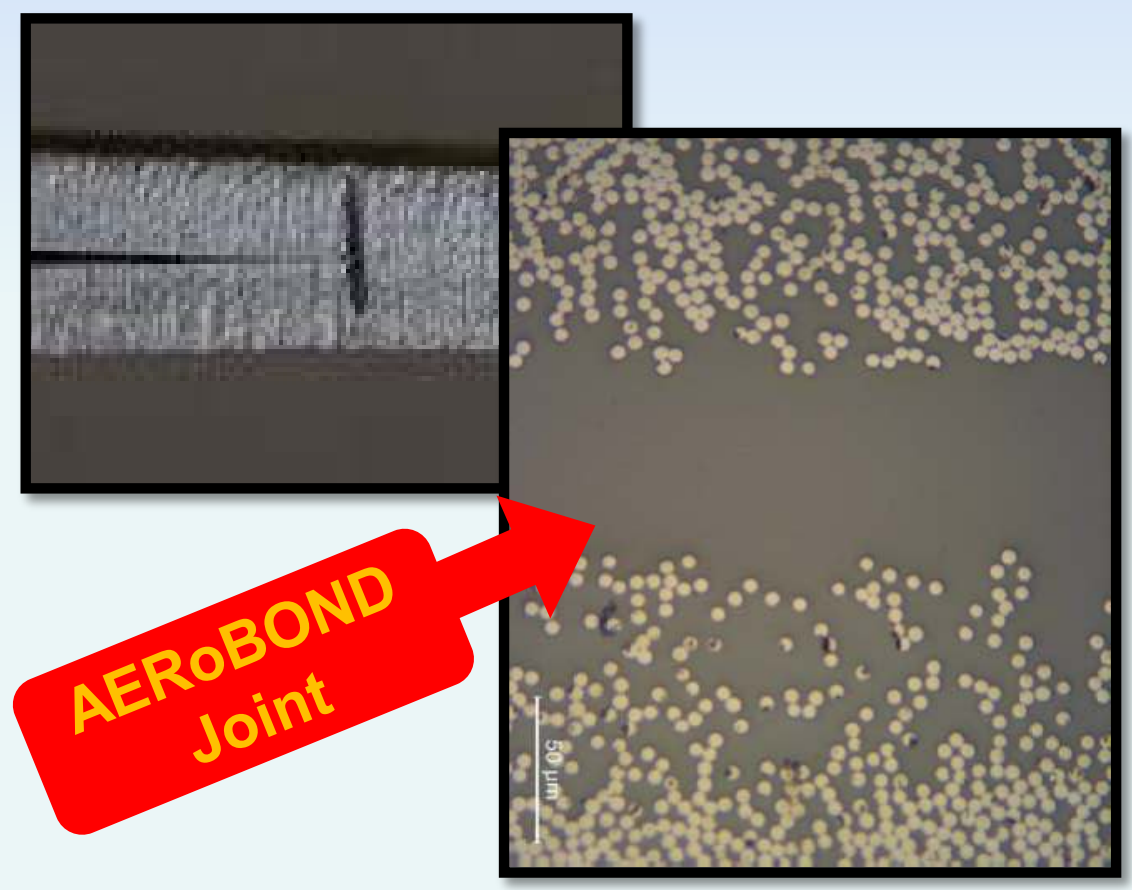

- The AERoBOND joint is indistinguishable from the matrix resin of adherends

Quantifiable, certifiable resin properties 


\section{Failure Mode and Certification}

\section{Bond Failure}
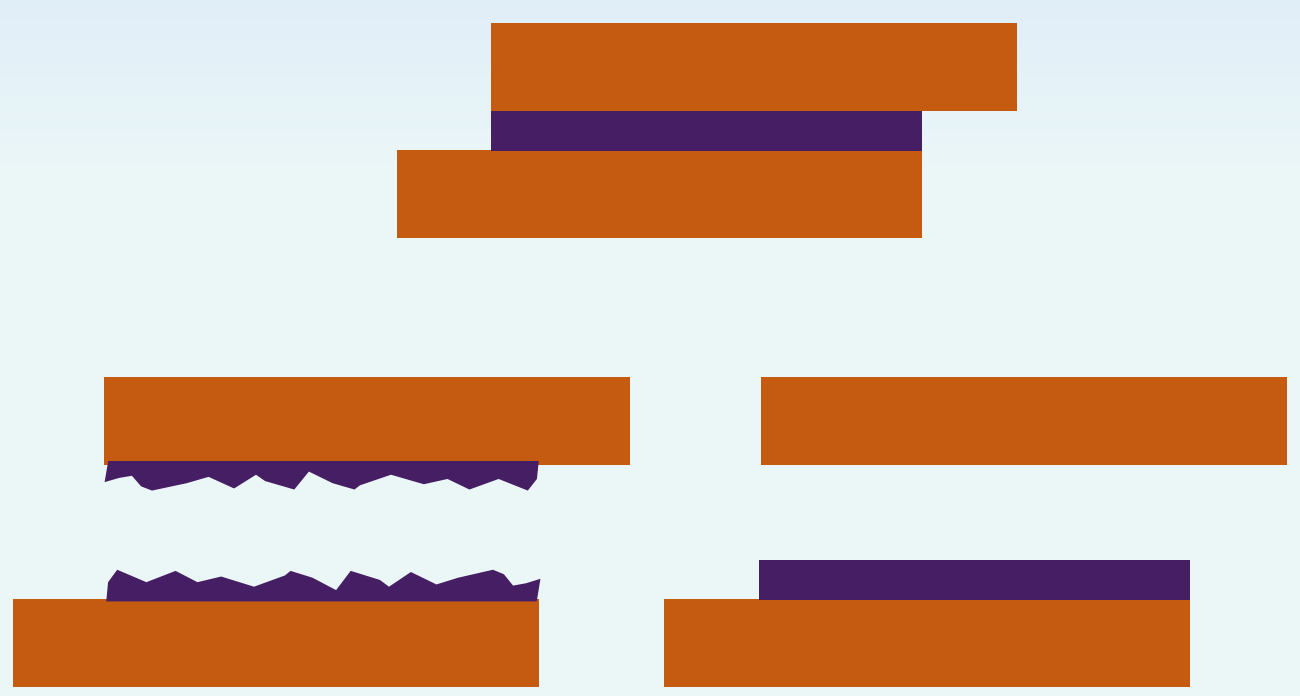

Cohesive Failure

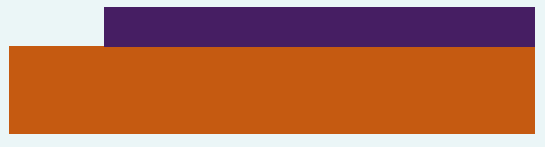

Adhesive Failure

\section{AERoBOND Failure}
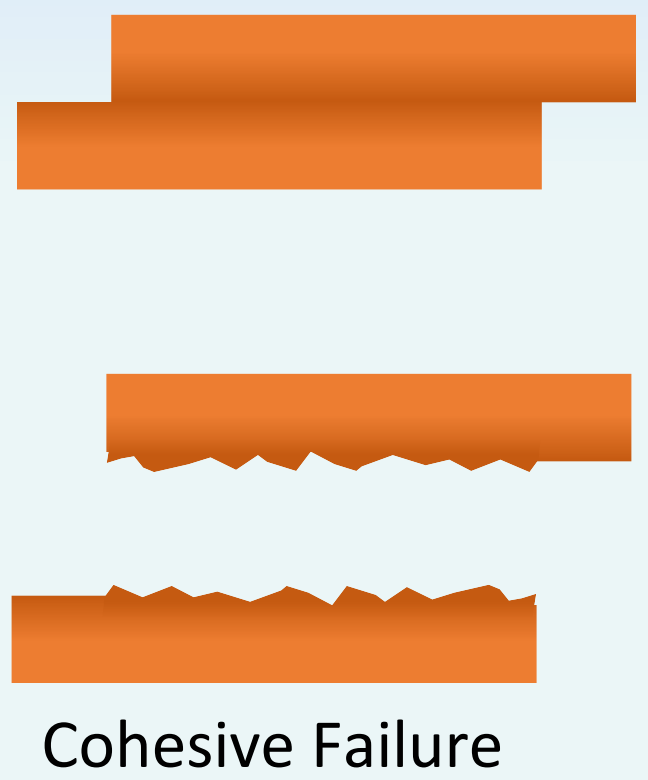

- AERoBOND eliminates the potential for weak bond failure mode

- Goal: AERoBOND mechanical properties similar to conventional co-cured laminates. 


\section{Thermoset Epoxy Basics}

- Hardener groups $(\mathrm{H})$ react with epoxy groups (E) to form polymer.

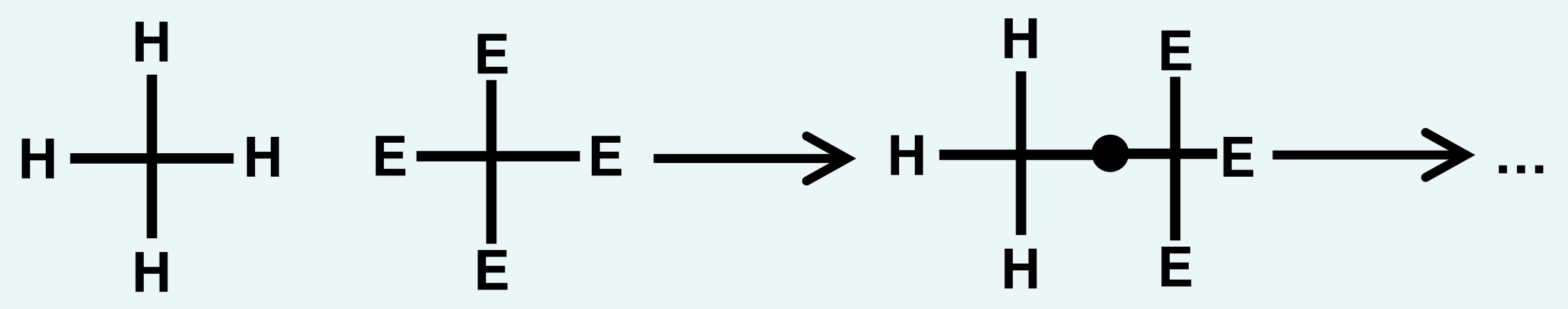

- Molecular weight is limited by applying an offset to the stoichiometry

$$
\frac{\text { hardener }}{\text { epoxy }}=r=\frac{\text { moles } H}{\text { moles } E} \quad \begin{aligned}
& r=1 \text { : Equivalent Mixture } \\
& r>1 \text { : Hardener Rich }(\mathrm{HR}) \\
& r<1: \text { Epoxy Rich (ER) }
\end{aligned}
$$




\section{Resin Chemistry}

- Kaneka API-60 Part A

- $\sim 65 \%$ tetrafunctional epoxy

- $20 \%$ trifunctional epoxy

- $15 \%$ proprietary toughener

- Epoxy equivalent weight known! $\mathrm{EEW}=131 \mathrm{~g} / \mathrm{mol}$

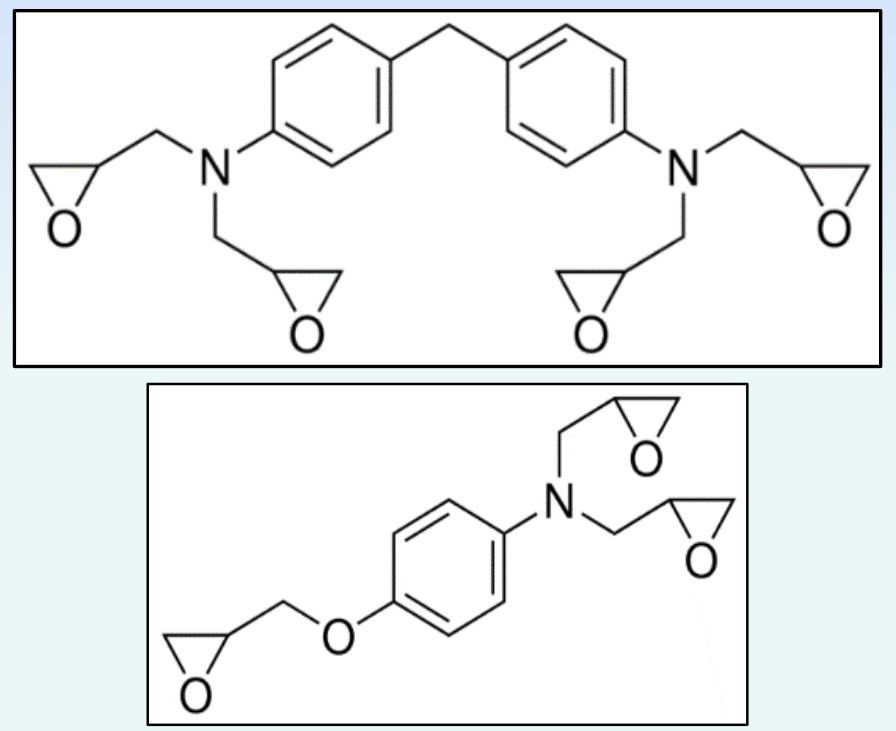

- Diethyltoluenediamine (DETDA)

- Ethacure ${ }^{\circledR} 100$ from Albemarle ${ }^{\circledR}$ Corp.

- Mixture of isomers

- Liquid at RT

- Equivalent weight, EW = $44.6 \mathrm{~g} / \mathrm{mol}$

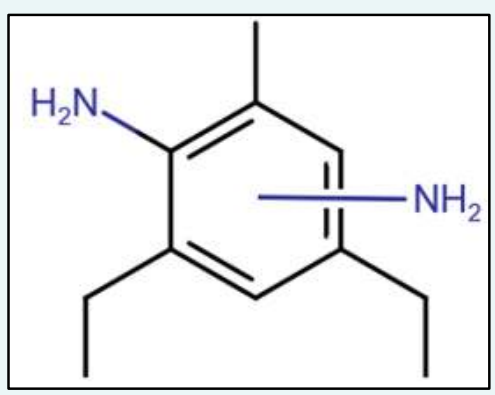




\section{Resin Preparation}

- API-60 Part A and DETDA stirred $1-2.5 \mathrm{~h}$ at $100-110{ }^{\circ} \mathrm{C}$

- Prepolymer diluted with 15 wt.\% methyl ethyl ketone (MEK) prior to producing prepreg
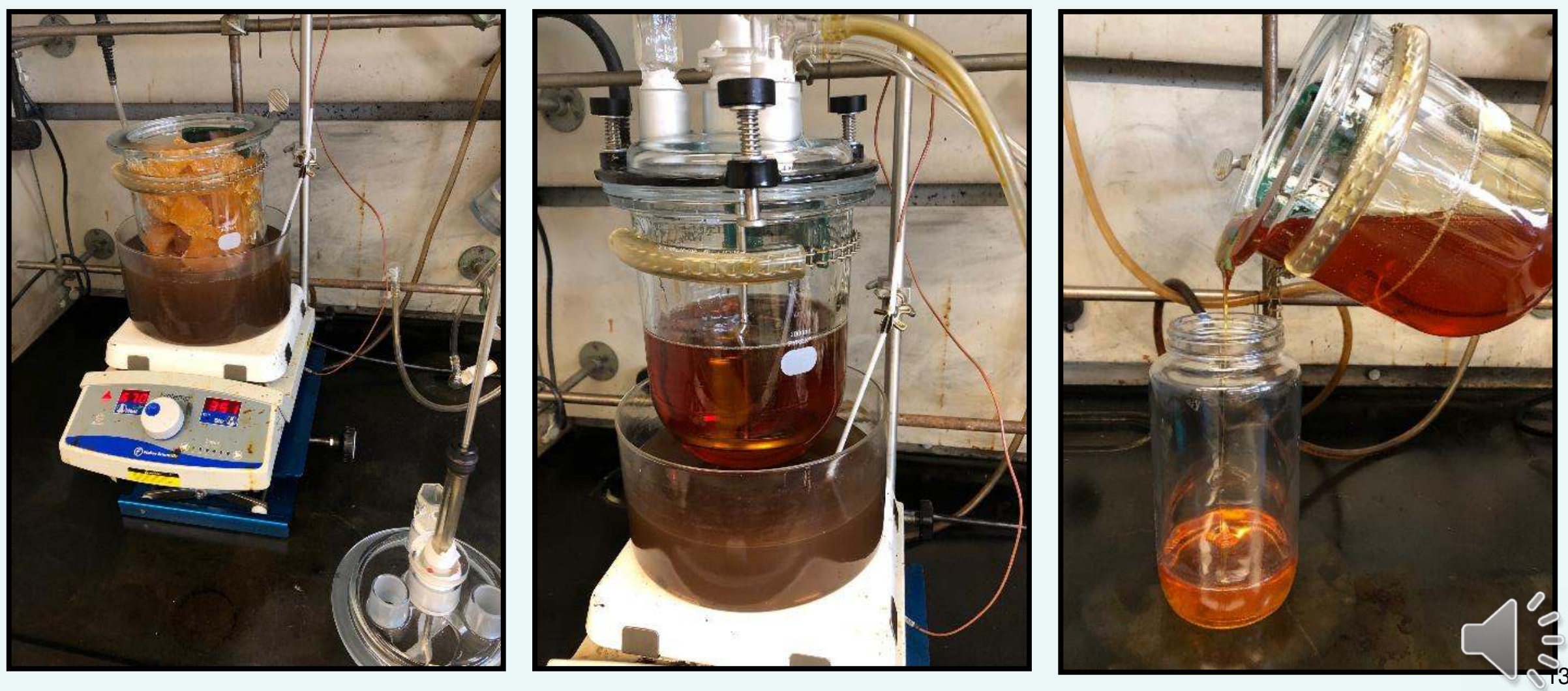


\section{Prepreg Tape Production}

- Prepared prepreg tape using a custom tape machine at NASA Langley Research Center

- Fiber: IM7G 12k, 14 to 16 tows, 75-100 mm wide tape

- Better uniformity and larger batches compared to hand painted film

- Process development is complex/challenging
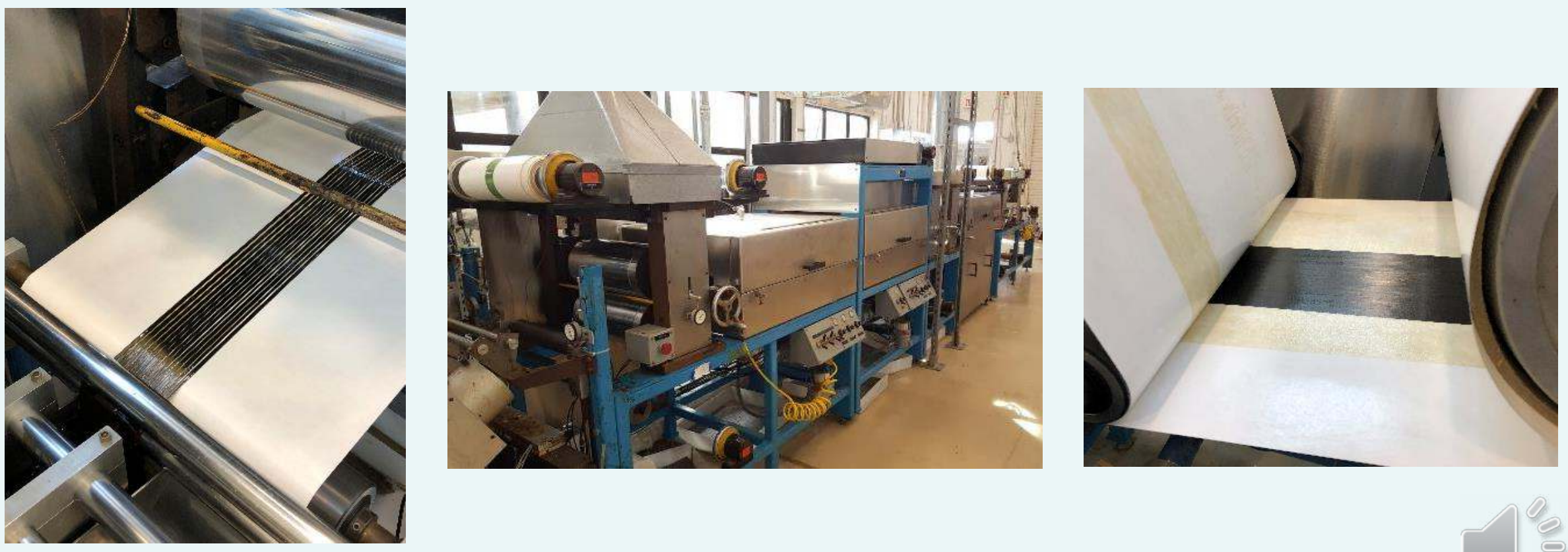


\section{Manufacturing Panels}

- Offset prepreg on conventional prepreg

- Panels cured in autoclave

Preform

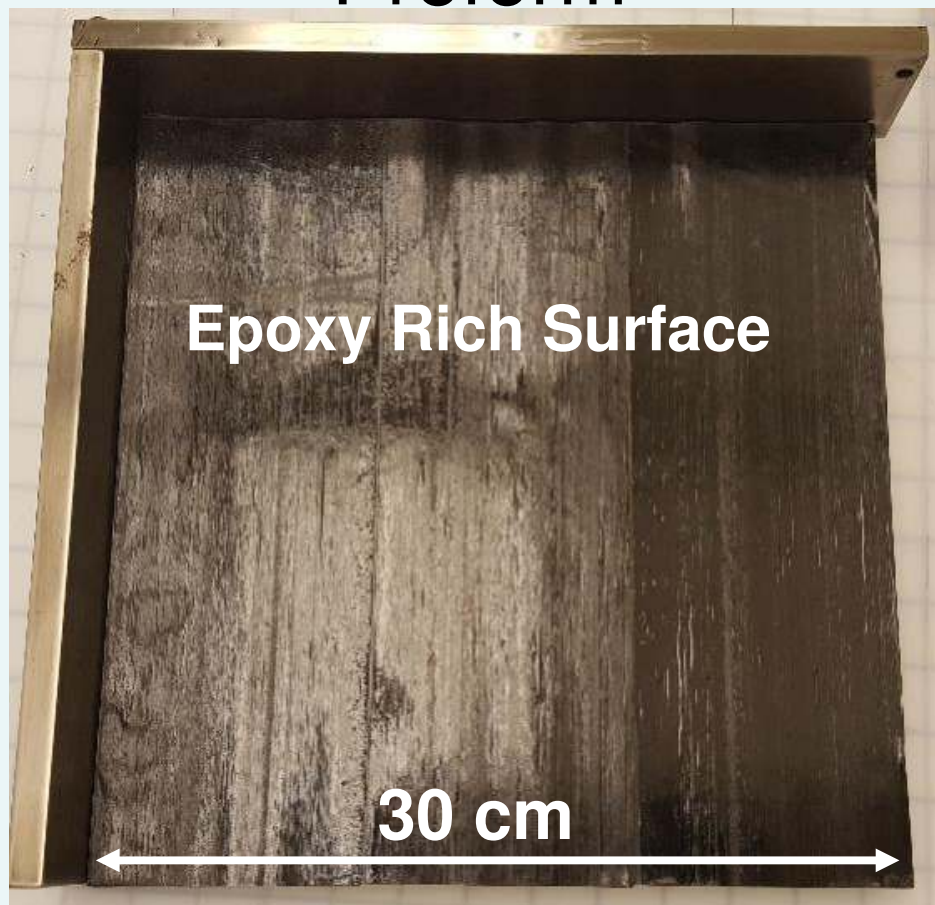

Cured Half-Panel

Epoxy Rich Surface 


\section{Preliminary Development and Testing}

- Two-step fabrication process

- 1. Fabricate "half-panels" with ER surfaces

- 2. Join ER panels with HR "adhesive" ply

- Conventional material used in backer laminate

- Hexcel ${ }^{\circledR} \mid \mathrm{M} 7 / 8552$ prepreg

- $190 \mathrm{~g} / \mathrm{m}^{2}$ fiber areal weight (FAW), 35\% resin content

Laminate Configuration

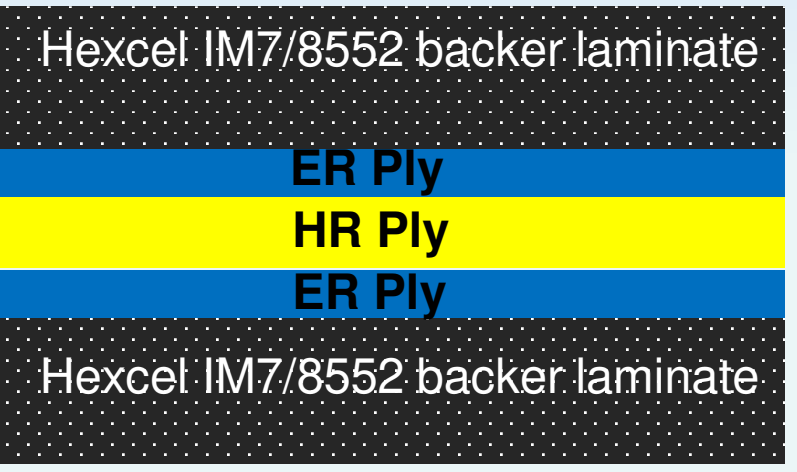

- Test Parameters

- Resin formulation ( $r$-value, degree of cure)

- Ply thickness \& resin content

End-Notched Flexure

- Cure process (time and temperature)

- Bagging scheme

- Rapid Screening with End-Notched Flexure (ENF) test

- Mode II (Shear) Fracture Toughness

- Simple specimen fabrication and testing

(ENF) Test

Fiber direction

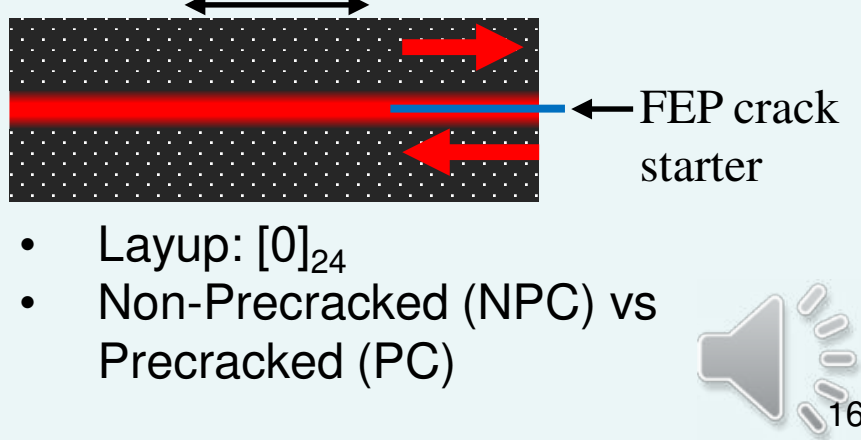




\section{Process Development}

\begin{tabular}{|c|c|c|}
\hline 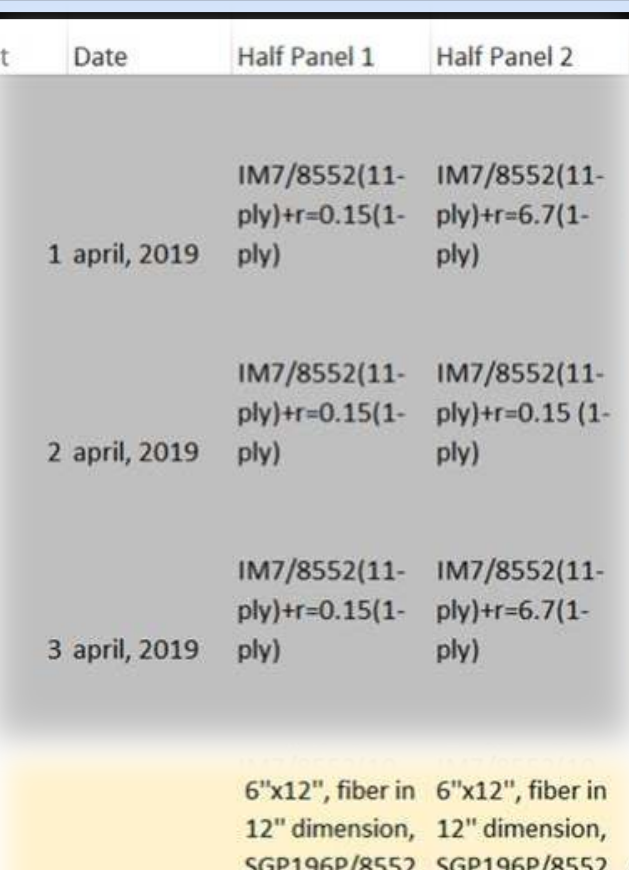 & $=$ & $\begin{array}{l}\text { Completed testing on } 30+ \\
\text { AERoBOND configurations } \\
\text { using } 18+\text { material systems } \\
\text { - End-notched flexure } \\
\text { - Failure locus, hardness } \\
\text { - Chemical analysis } \\
\text { Each configuration spans } 3+ \\
\text { weeks of effort }\end{array}$ \\
\hline 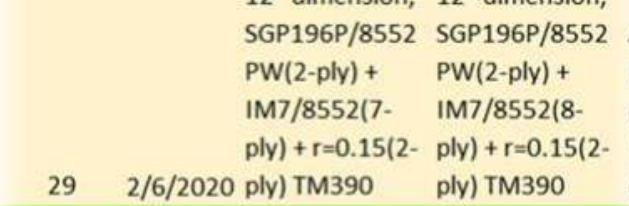 & & 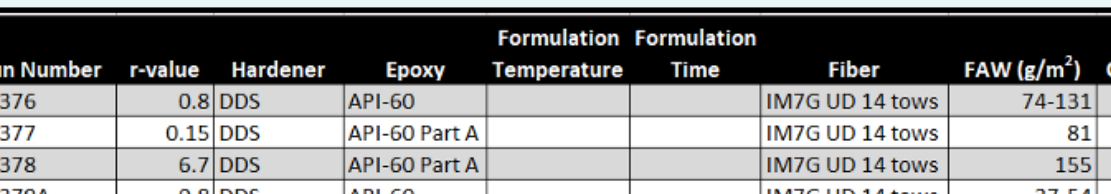 \\
\hline & & \\
\hline
\end{tabular}




\section{Some Vague Details}

\begin{tabular}{|l|l|l|l|l|l|}
\hline Material & $\boldsymbol{r}$-value & FAW $\left(\mathbf{g} / \mathbf{m}^{2}\right)$ & RAW $\left(\mathbf{g} / \mathbf{m}^{2}\right)$ & $\begin{array}{l}\text { Number } \\
\text { of Plies }\end{array}$ & $\begin{array}{l}\text { Primary Cure } \\
\text { Cycle }\end{array}$ \\
\hline $\begin{array}{l}\text { ER Ply } \\
\text { Ranges }\end{array}$ & 0.15 & $37-90$ & $60-125$ & $1-2$ & $1-3 \mathrm{~h} \mathrm{@} 177^{\circ} \mathrm{C}$ \\
\hline $\begin{array}{l}\text { HR Ply } \\
\text { Ranges }\end{array}$ & $2.5-6.7$ & $56-155$ & $20-90$ & $1-2$ & $1-4 \mathrm{~h} @ 177^{\circ} \mathrm{C}$ \\
\hline $\begin{array}{l}\text { Round 1 ER } \\
\text { Round 1 HR }\end{array}$ & 0.15 & 77 & 150 & 2 & $1 \mathrm{~h} @ 177^{\circ} \mathrm{C}$ \\
\hline R & 70 & $225^{\mathrm{B}}$ & 1 & $4 \mathrm{~h} @ 177^{\circ} \mathrm{C}$ \\
\hline
\end{tabular}

A. Stoichiometric offset to $r=2.5$ does not prevent gelation, HR ply only sees $2^{\text {nd }}$ cure cycle

B. Hand painted resin onto carrier scrim cloth to achieve higher resin loading 


\section{Key Preliminary Results}

- Experiment 15 exceeded expectations (90\% of baseline)

- But successful results were difficult to repeat

- Used hand painted resin film for HR ply

- Experiment 20 was also interesting (50\% of baseline)

- Also prepared from a large batch of hand painted film

- Used configuration from experiment 20 for Round 1 samples
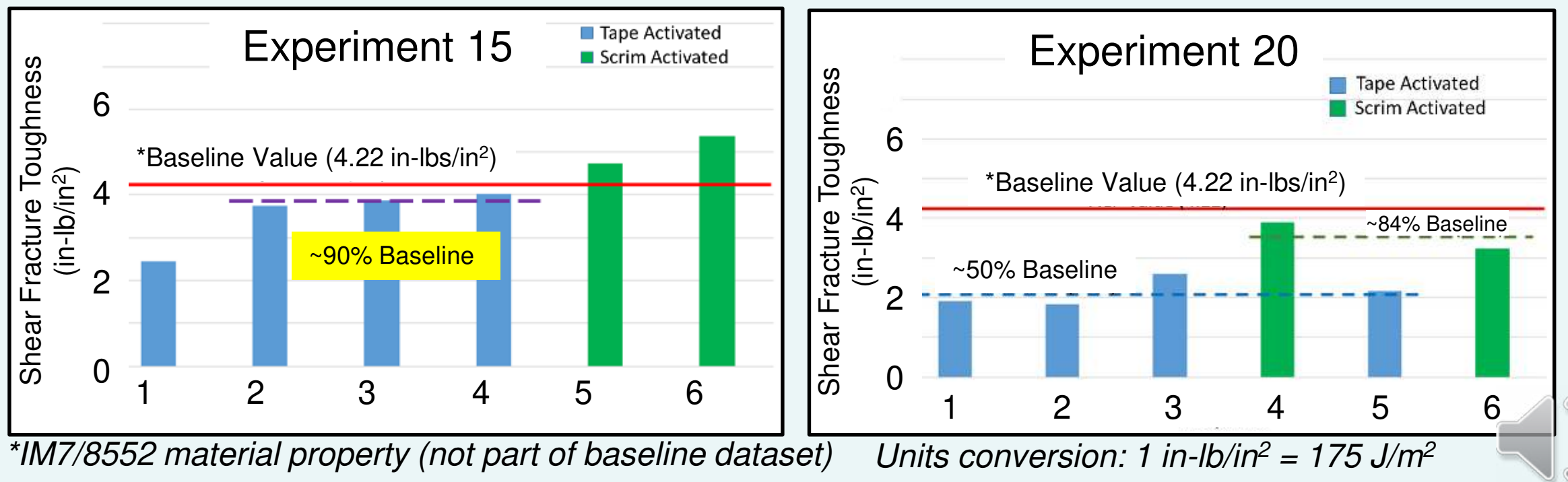


\section{Baseline Properties}

- Measured properties for a series of laminates fabricated using:

- AERoBOND materials (epoxy and hardener)

$-r=0.8$ (conventional ratio)

- Co-cure process

- Baseline properties used to set AERoBOND performance goals 


\section{End-Notched Flexure (ENF) Test}

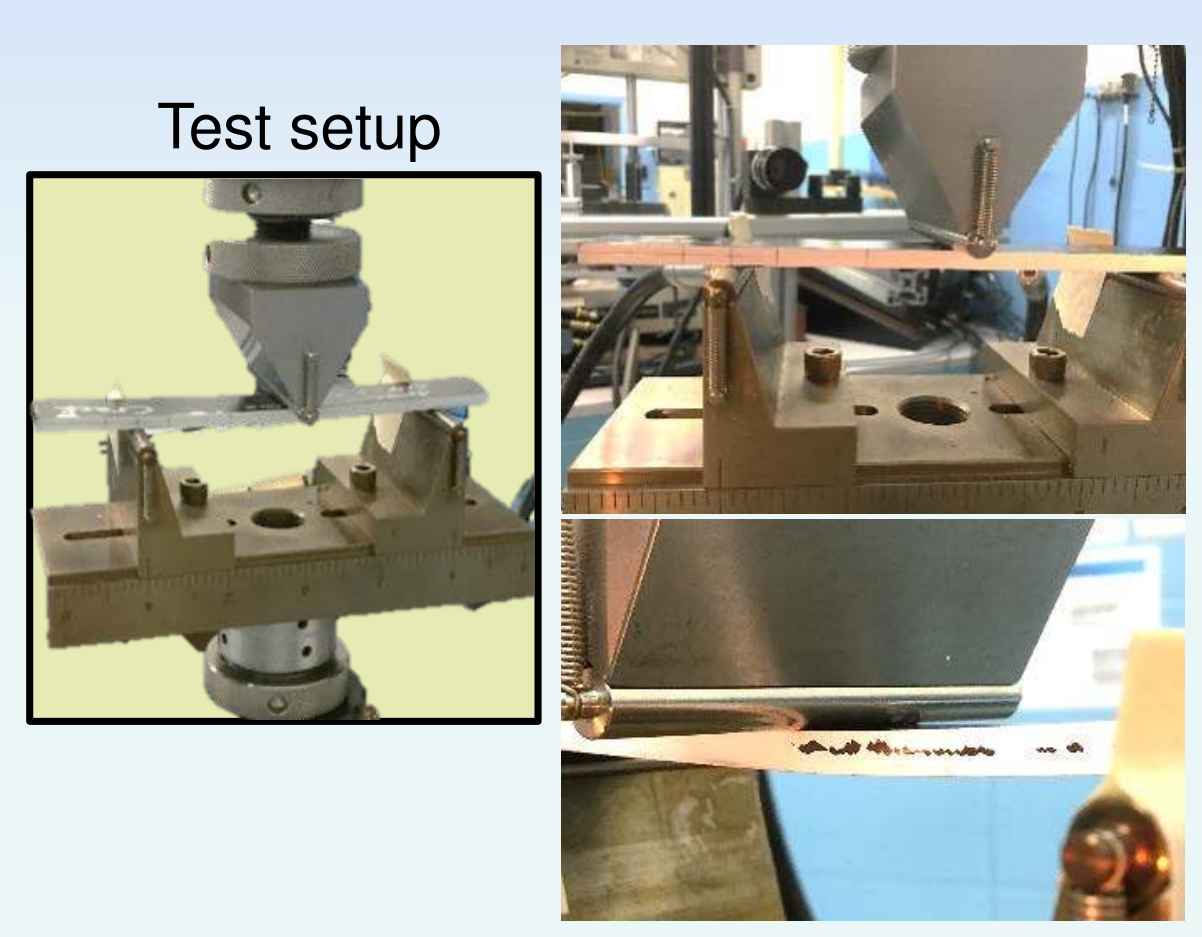

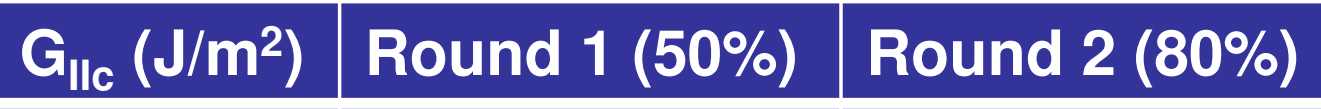

Goals*

370

591

Measured $\quad 347 \pm 97$ (47\%)

*Based on precracked value measured on IM7/8552
Mode-II Interlaminar Fracture Toughness

Fiber direction

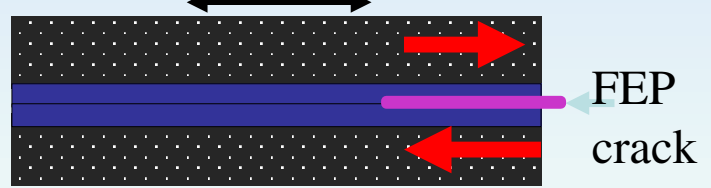

- Layup: $[0]_{24}$
Large amount of data scatter due to multiple failure mechanisms but close to $50 \%$ goal.

Baseline Properties

${ }^{*}$ Ave $G_{\| c-P C}: 740 \mathrm{~J} / \mathrm{m}^{2}$

*Std Dev: $\quad 50.4 \mathrm{~J} / \mathrm{m}^{2}$

${ }^{*}$ Cof Var: $\quad 6.8 \%$ 
Double Cantilever Beam (DCB) Test Mode I Fracture Toughness

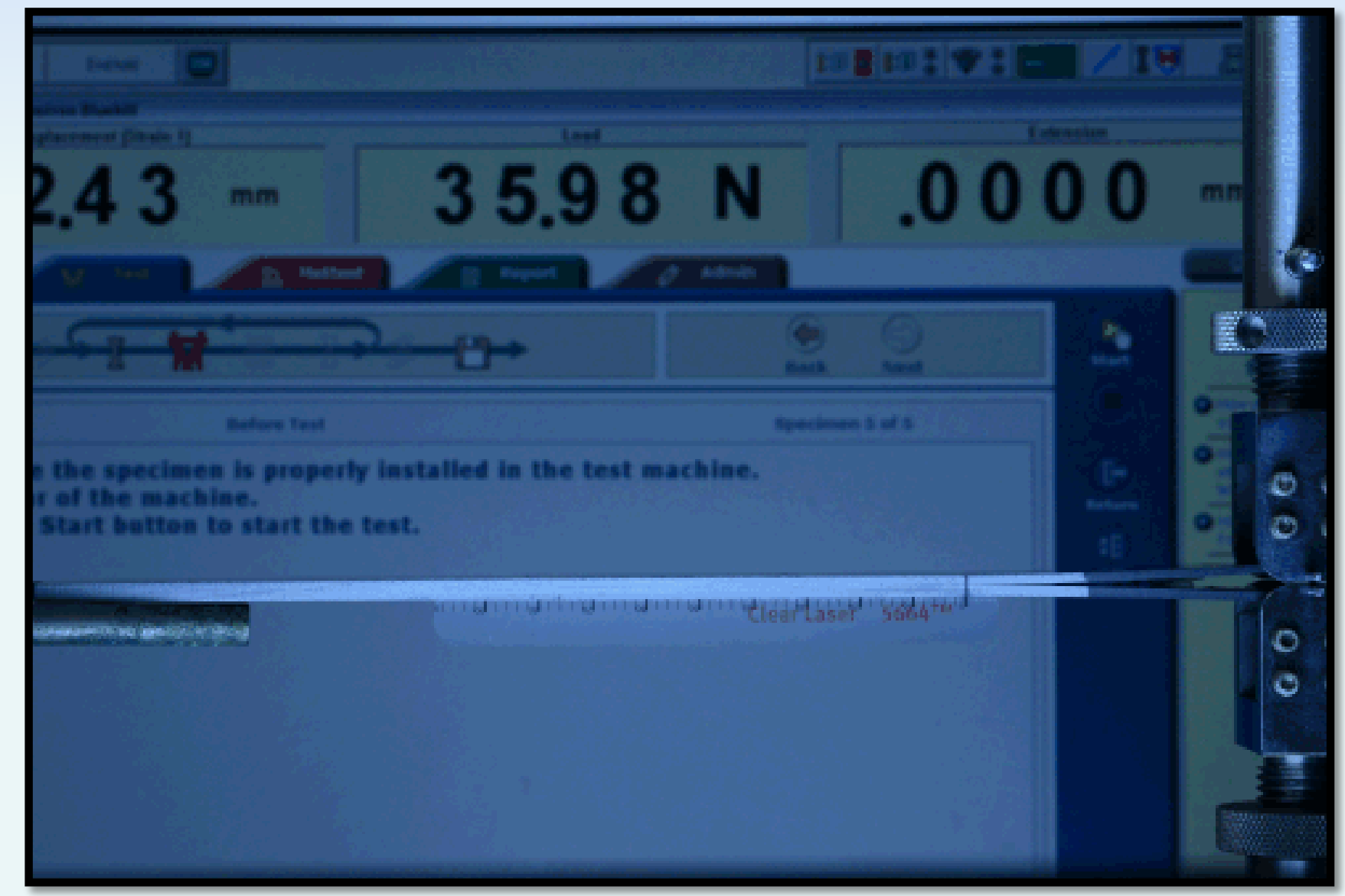




\section{Summary of Joint Properties}

\begin{tabular}{|c|c|c|c|c|}
\hline Strength & $\begin{array}{c}\text { ILTCB } \\
(\mathrm{MPa})\end{array}$ & $\begin{array}{c}\text { ILTFWT } \\
(\mathrm{MPa})\end{array}$ & $\begin{array}{c}\text { ILS } \\
(\mathrm{MPa})\end{array}$ & $\begin{array}{c}\text { ILS }^{\mathrm{DLS}} \\
(\mathrm{MPa})\end{array}$ \\
\hline Baseline & 61.5 & 41.8 & 31.4 & 41.6 \\
\hline Round 1 & $23.5 \pm 2.2$ & 37.5 & $25.7 \pm 2.2$ & $9.85 \pm 1.5$ \\
\hline$\%$ of Baseline & $41 \%$ & $59 \%$ & $82 \%$ & $24 \%$ \\
\hline
\end{tabular}

Interlaminar Tensile (ILT), Curved Beam (CB), Flatwise Tension (FWT), Single-Lap Shear (SLS), Double-Lap Shear (DLS)

\begin{tabular}{|c|c|c|c|c|}
\hline $\begin{array}{c}\text { Fracture } \\
\text { Toughness }\end{array}$ & $\begin{array}{c}\mathrm{G}_{\mathrm{Ic} \text { init }} \\
\left(\mathrm{J} / \mathrm{m}^{2}\right)\end{array}$ & $\begin{array}{c}\mathrm{G}_{\mathrm{Ic} \_ \text {ss }} \\
\left(\mathrm{J} / \mathrm{m}^{2}\right)\end{array}$ & $\begin{array}{c}\mathrm{G}_{\text {Ilc_NPC }} \\
\left(\mathrm{J} / \mathrm{m}^{2}\right)\end{array}$ & $\begin{array}{c}\mathrm{G}_{\text {Ilc_PC }} \\
\left(\mathrm{J} / \mathrm{m}^{2}\right)\end{array}$ \\
\hline Test method & DCB & DCB & ENF & ENF \\
\hline Baseline & 180 & 203 & 1255 & $740^{*}$ \\
\hline Round 1 & $16 \pm 3.6$ & $36 \pm 16$ & $372 \pm 99$ & $347 \pm 97$ \\
\hline$\%$ of Baseline & $9 \%$ & $19 \%$ & $27 \%$ & $47 \%$ \\
\hline
\end{tabular}

Double Cantilever Beam (DCB), End-Notched Flexure (ENF), Critical strain energy release rate for mode-I $\left(\mathrm{G}_{\mathrm{IC}}\right)$ initiation (init) and steady-state (ss), and mode-II ( $\left.\mathrm{G}_{\| \mathrm{lc}}\right)$ non-precracked (NPC), and precracked (PC) */M7/8552 material property (not part of baseline dataset) 


\section{Progress Since Round 1 (G $\left.G_{I I C \_N P C}\right)$}

- Recent tests improved over Round 1 (27\%) and even exceeded baseline toughness

- Baseline $\pm \sigma$ indicated with red bar

- $50 \%$ baseline indicated with orange line

- ER activated (blue bars) $\mathbf{1 1 1 \%}$ of baseline

- HR activated (green bars) $64 \%$ of baseline

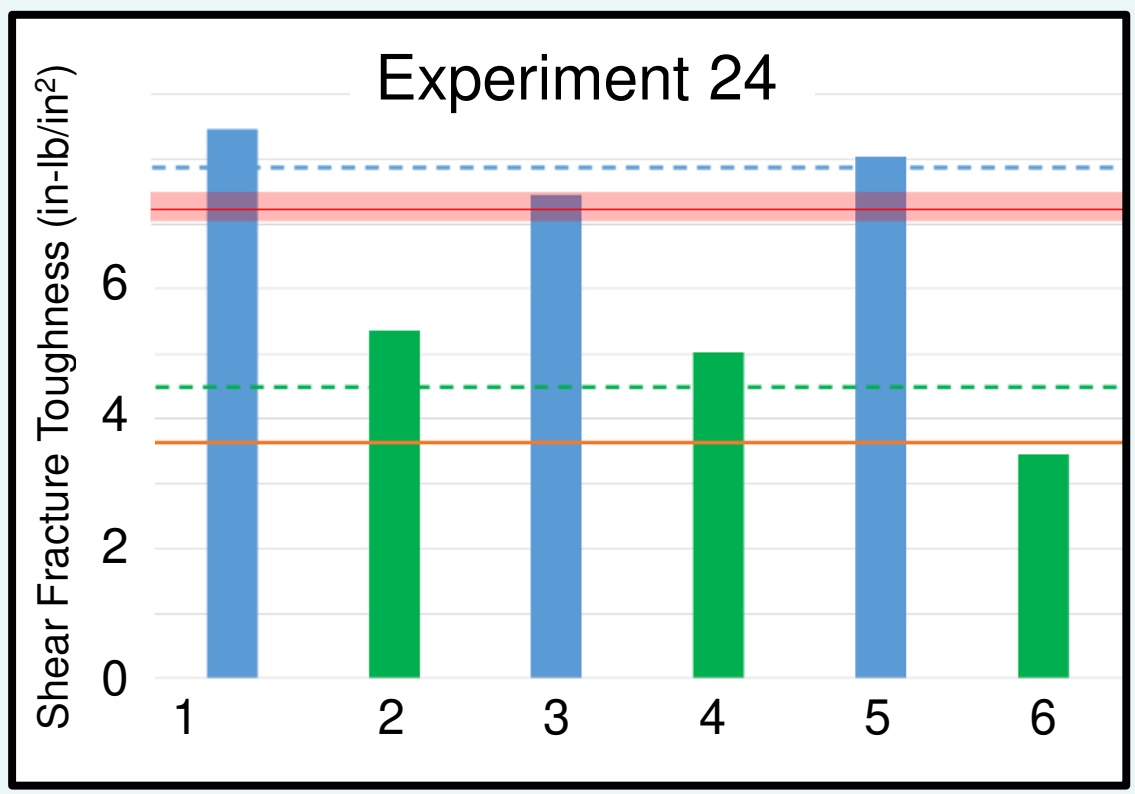

Ave: 7.496 in-lbf/in² $\%$ Baseline: $105 \%$

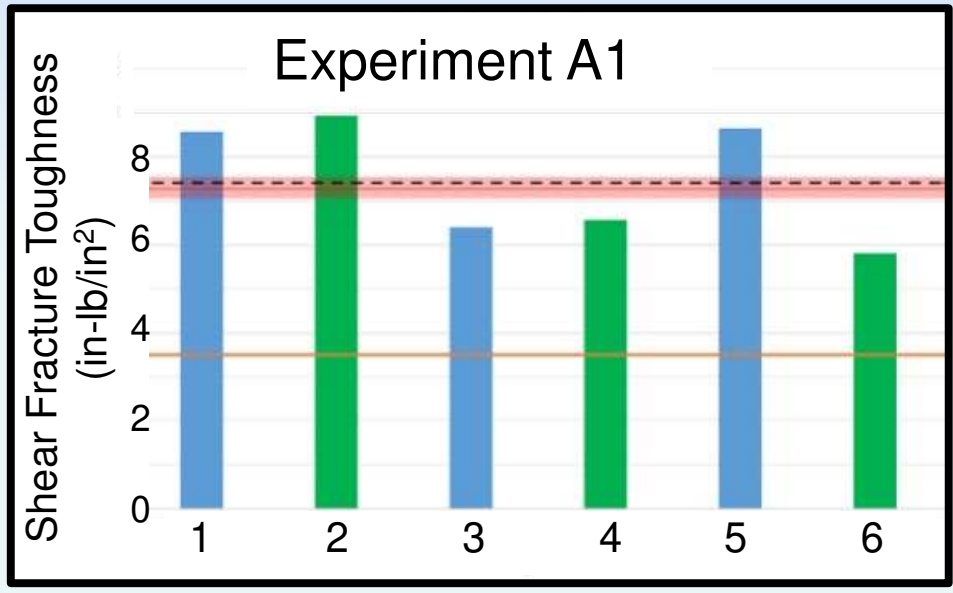

Ave: 7.379 in-lbf/in² $\%$ Baseline: $103 \%$

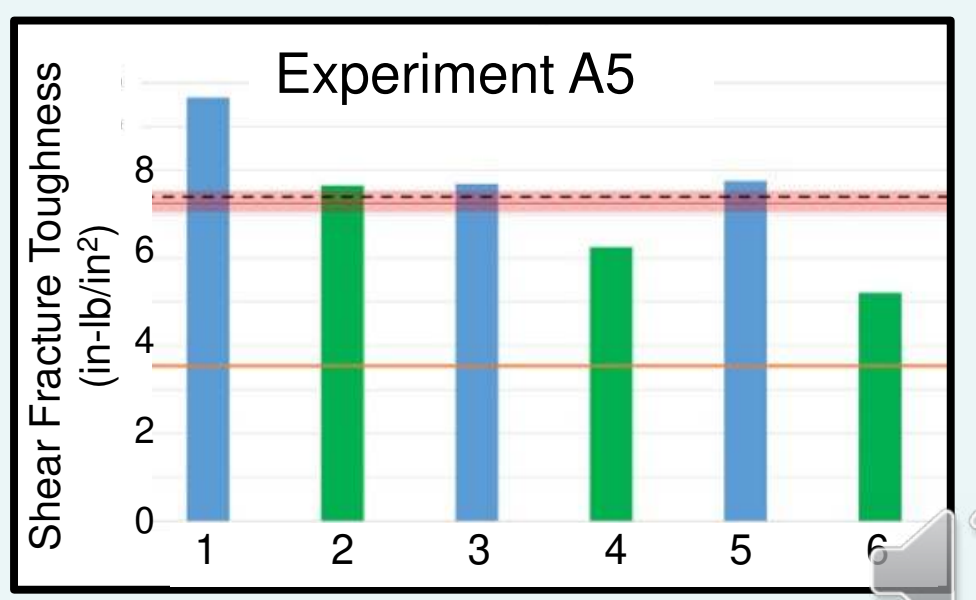

Units conversion: $1 \mathrm{in}-\mathrm{lb} / \mathrm{in}^{2}=175 \mathrm{~J} / \mathrm{m}^{2}$ 


\section{Progress Since Round 1 (G $\left.G_{\text {IIC_PC }}\right)$}

- Recent tests improved over Round 1 (47\%) and even exceeded baseline toughness

- Baseline indicated with red line

- $50 \%$ baseline indicated with orange line Greatly improved shear fracture toughness

Ave: 6.26 in-lbf/in ${ }^{2} \quad \% B_{a s e l i n e}$ : $148 \%$

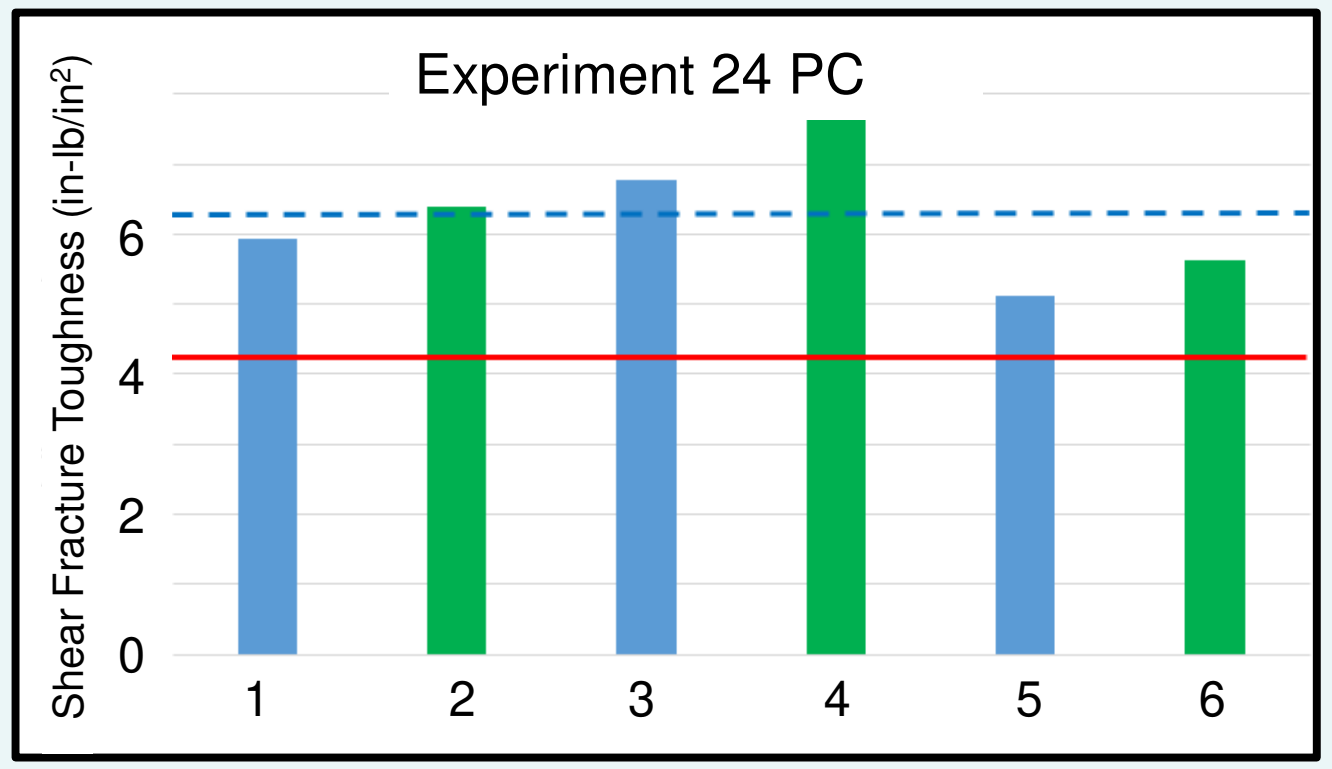

*IM7/8552 material property (not part of baseline dataset)

Unit conversion: $1 \mathrm{in}-\mathrm{lb} / \mathrm{in}^{2}=175 \mathrm{~J} / \mathrm{m}^{2}$

Ave: 4.79 in-lbf/in² \%Baseline*: 113\%

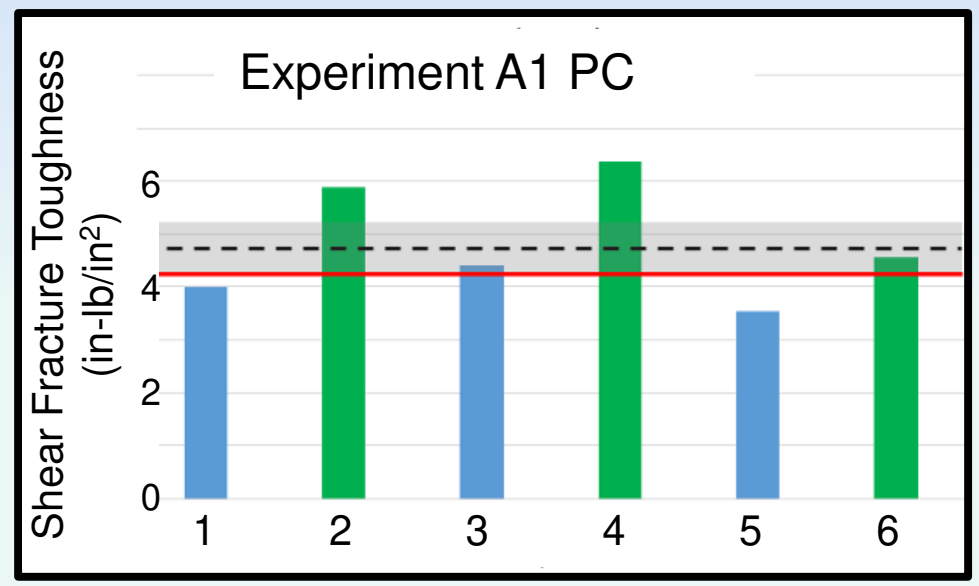

Ave: $5.410 \mathrm{in}-\mathrm{lbf} / \mathrm{in}^{2} \quad \%$ Baseline* $: 128 \%$

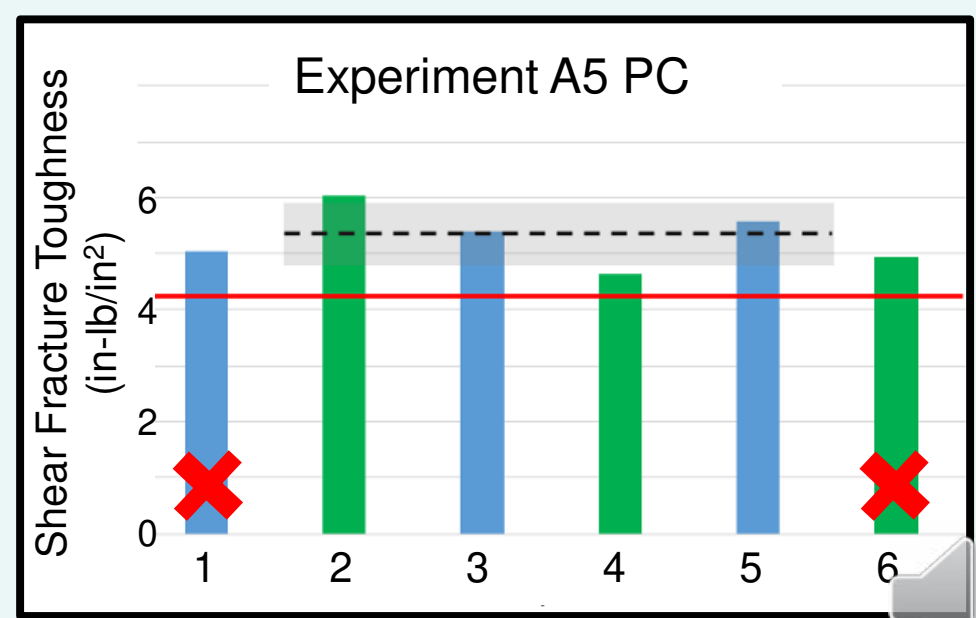




\section{Optical Inspection of Interface}

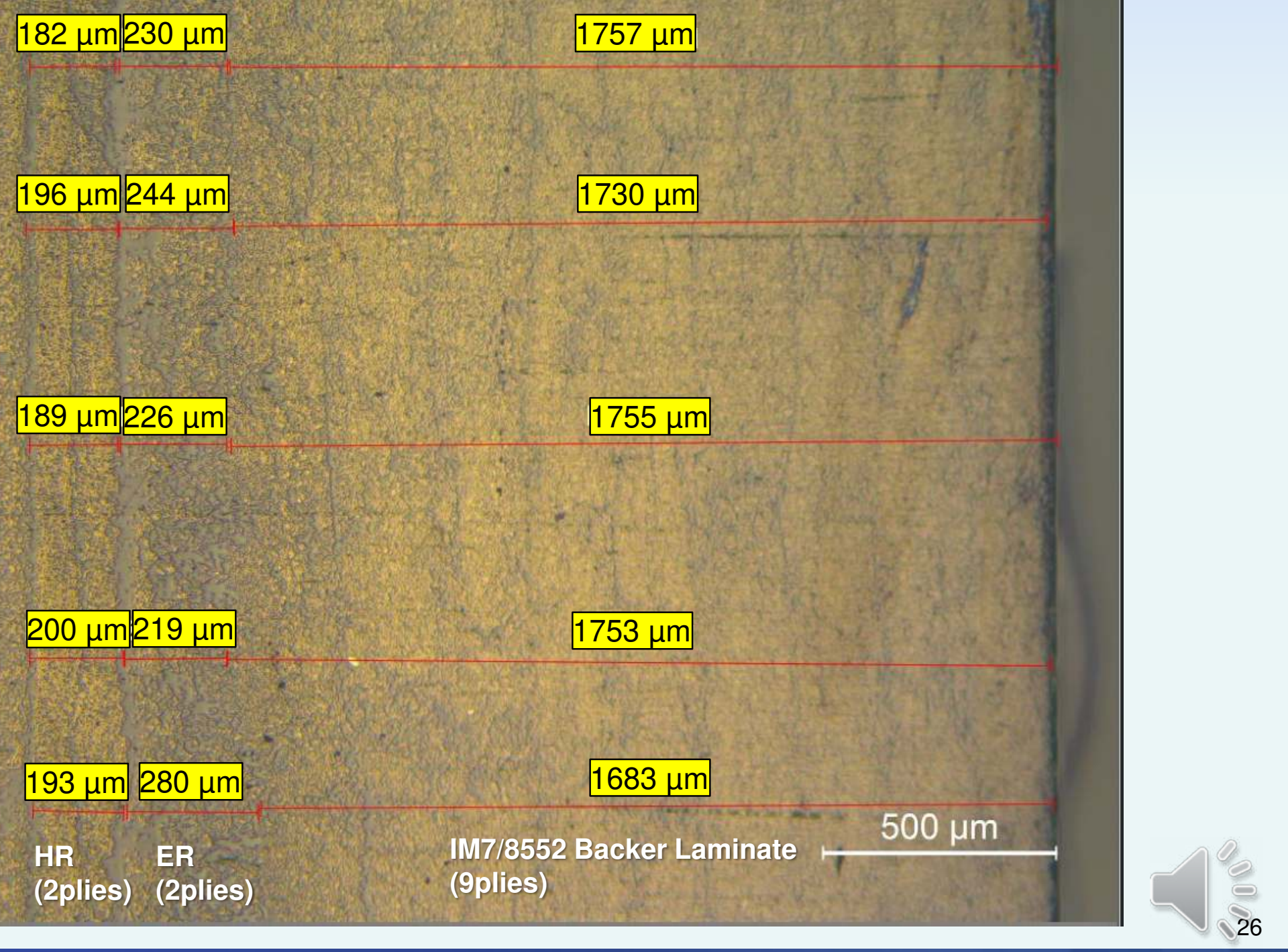




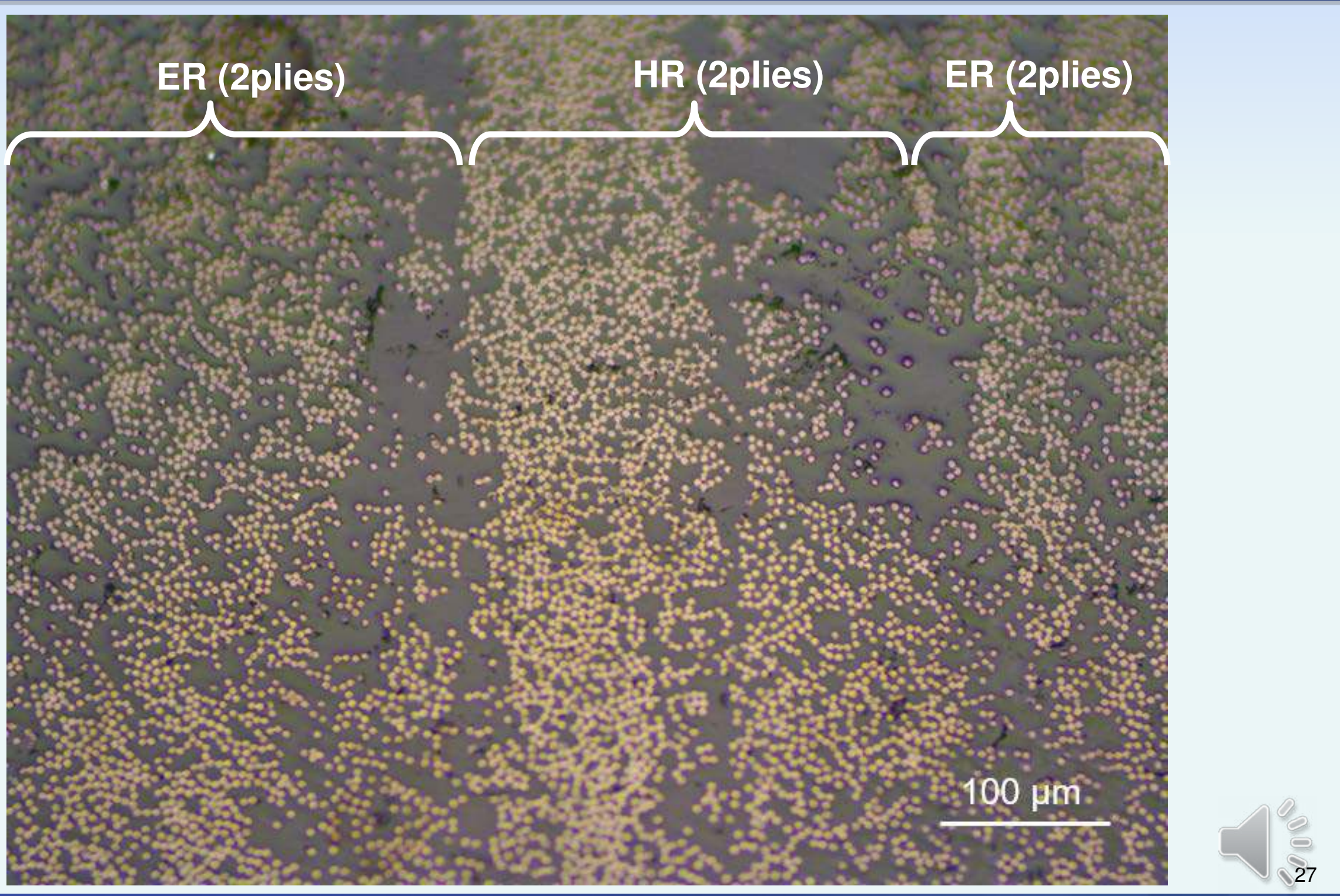




\section{Conclusions}

- AERoBOND approach:

- Achieve predictability of co-cured joints with the manufacturing simplicity of secondary bonding

- AERoBOND joint should be indistinguishable from interlaminar joint and similar in properties

- Microscopic inspection indicates good mixing at AERoBOND interface

- Several tests indicate we have reached our preliminary goal of $50 \%$ baseline properties

- Interlaminar shear (SLS) and tensile (FWT, CB) strengths

- Shear fracture toughness (ENF) is close to goal

- Recent results (ENF) indicate AERoBOND process can match co-cure properties 


\section{Acknowledgements}

- Glenn Research Center:

- Mechanical testing: Jon Salem \& Mike Pereira

- Langley Research Center:

- Engineering: James Ratcliffe

- Laminate fabrication: Sean Britton, Hoa Luong, \& Kelvin Boston

- Machining: Mike Oliver \& Thomas Hall 
ThankYou!

(frankl.palmieri@nasa.gov) 


\section{Start Extra Material}




\section{Mechanical Test Methods 1}

\section{Quasi-static testing}

- ASTM D5528 (DCB) \& D7905 (ENF) for mode I \& mode II interlaminar fracture toughness

- ASTM D3165 (SLS) \& D3528(DLS): Apparent shear strength

- ASTM D6415 (CB): Interlaminar tensile strength
Double Cantilever Beam (DCB) \& End-Notched Flexure (ENF)

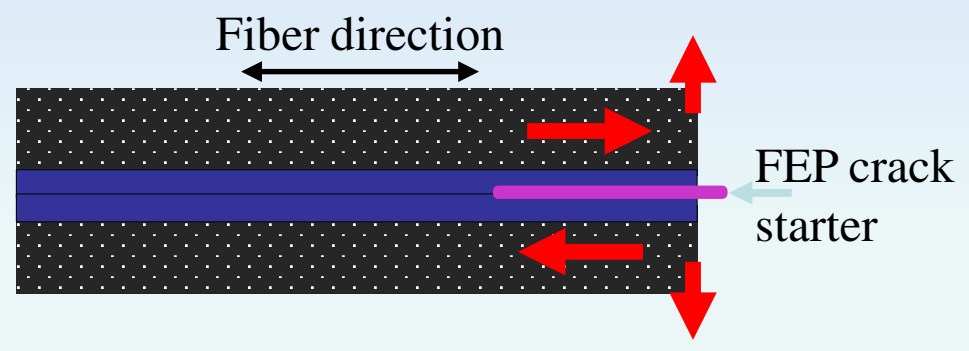

Single-Lap Shear (SLS) \& DoubleLap Shear (DLS)

Fiber direction

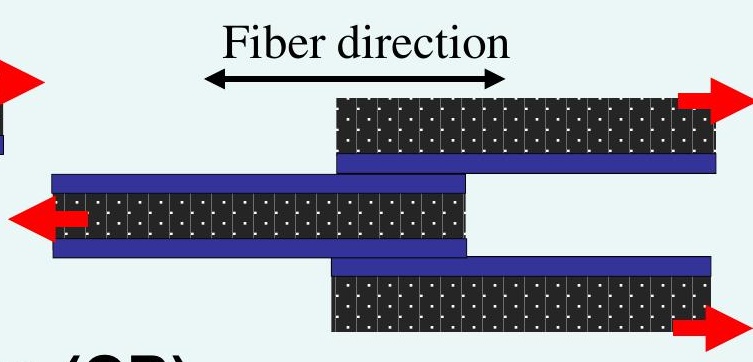

Curved Beam (CB)

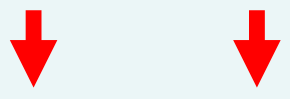




\section{Mechanical Test Methods 2}

Flatwise Tension (FWT)

- ASTM D7291:Interlaminar Tensile Strength
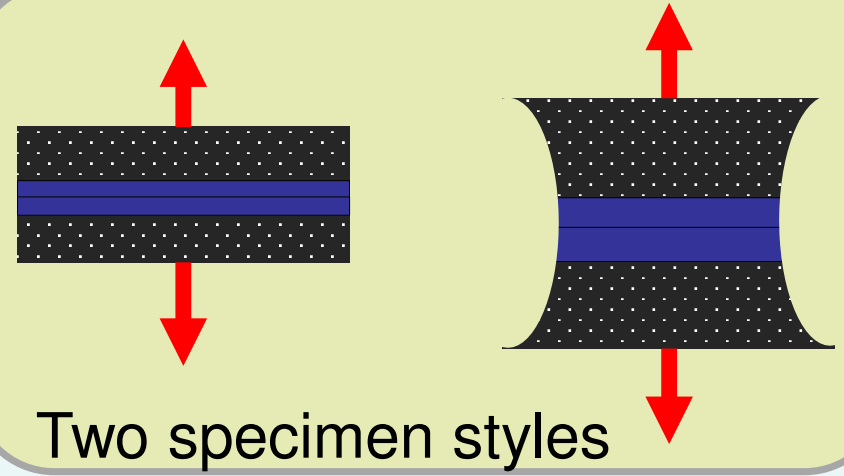

- ASTM D7136 (BVID) and

Two specimen styles ASTM D7137 (CAI):

Damage tolerance

Barely Visible Impact Damage (BVID) \& Compression After Impact (CAI)

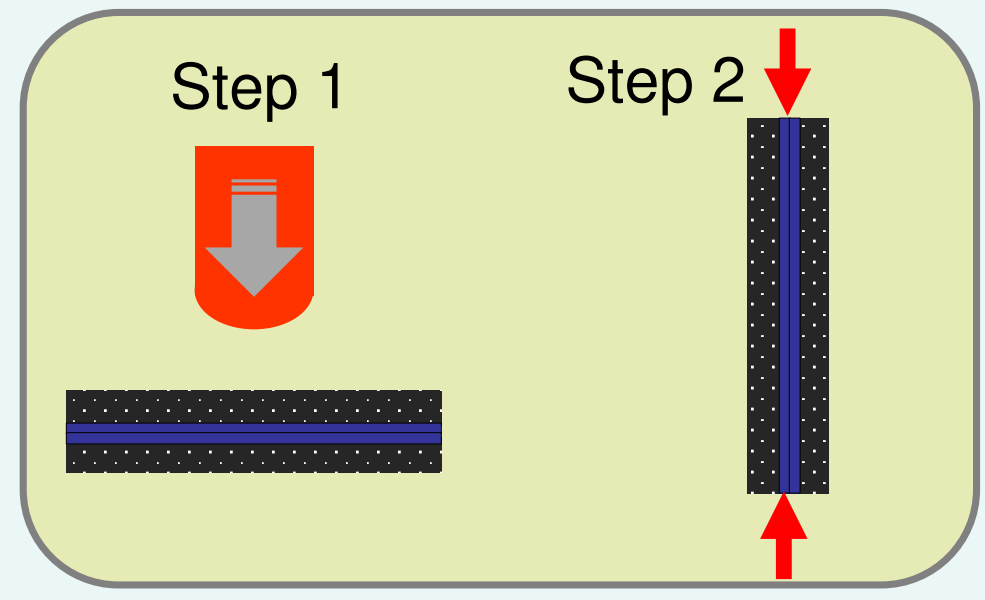




\section{Prepreger Details}
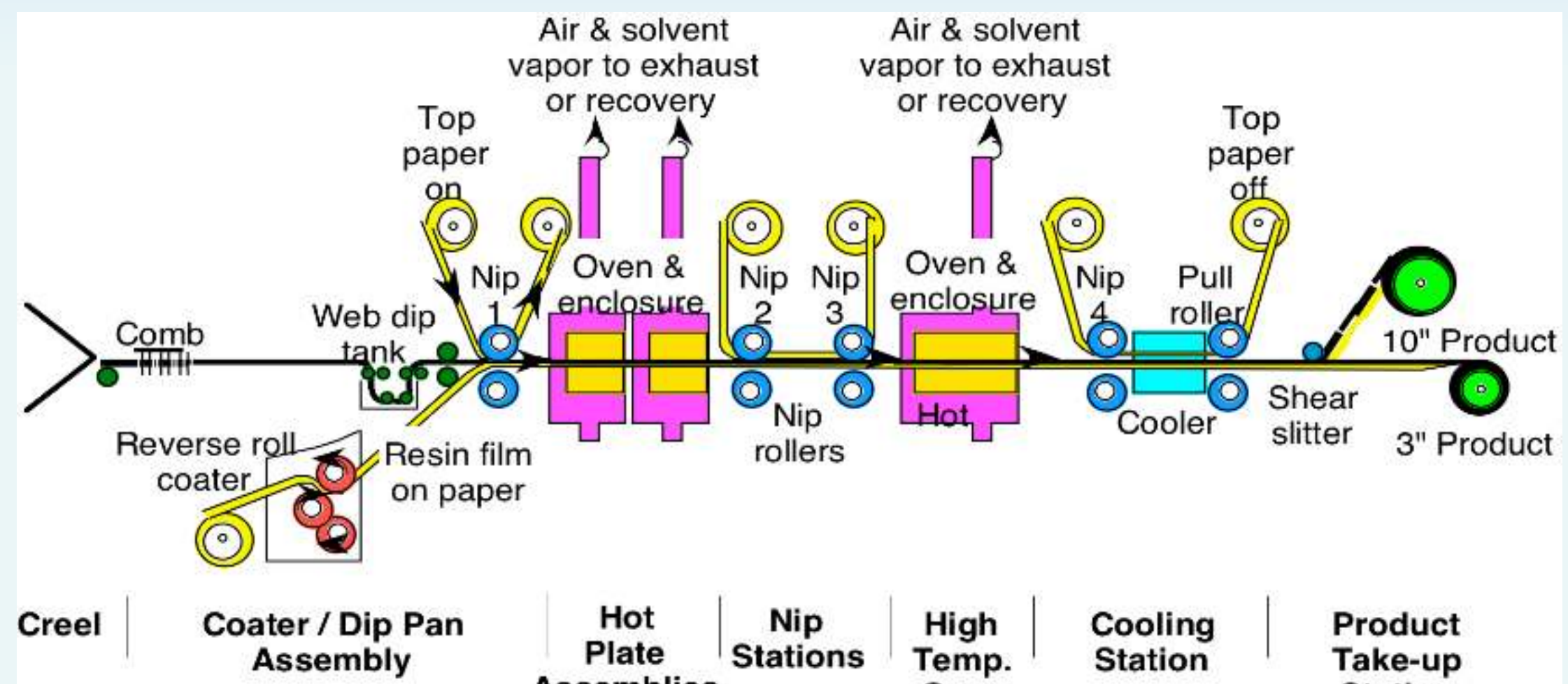

$$
\begin{array}{c|c|c|c}
\begin{array}{c}
\text { Hot } \\
\text { Plate }
\end{array} & \begin{array}{c}
\text { Nip } \\
\text { Stations }
\end{array} & \begin{array}{c}
\text { High } \\
\text { Temp. } \\
\text { Oven }
\end{array} & \begin{array}{c}
\text { Cooling } \\
\text { Station }
\end{array} \\
\text { Assemblies } &
\end{array}
$$

Product

Take-up

Station 


\section{2) EDS Map of Interface}

F K $\alpha 1 \_2$

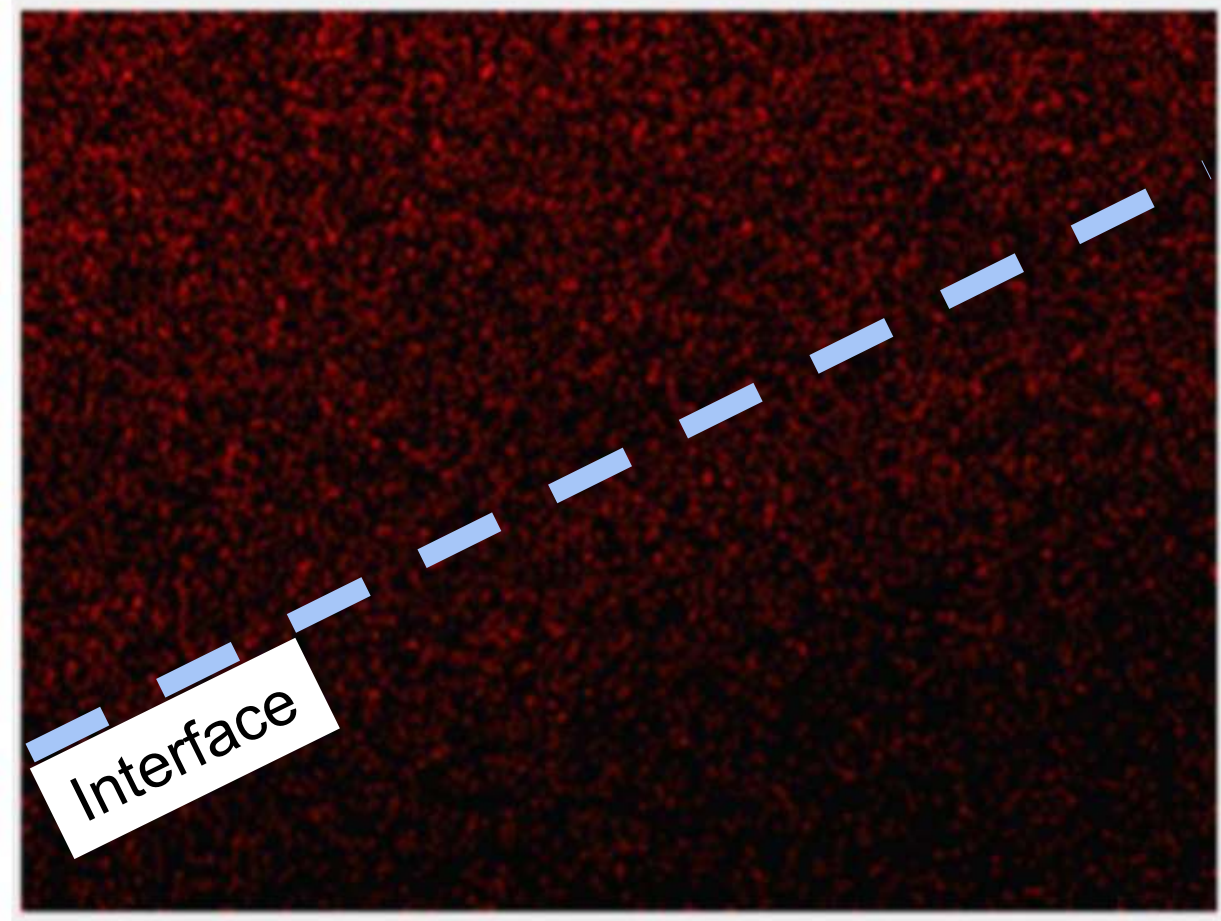

$100 \mu \mathrm{m}$

\section{$\mathrm{S} K \alpha 1$}

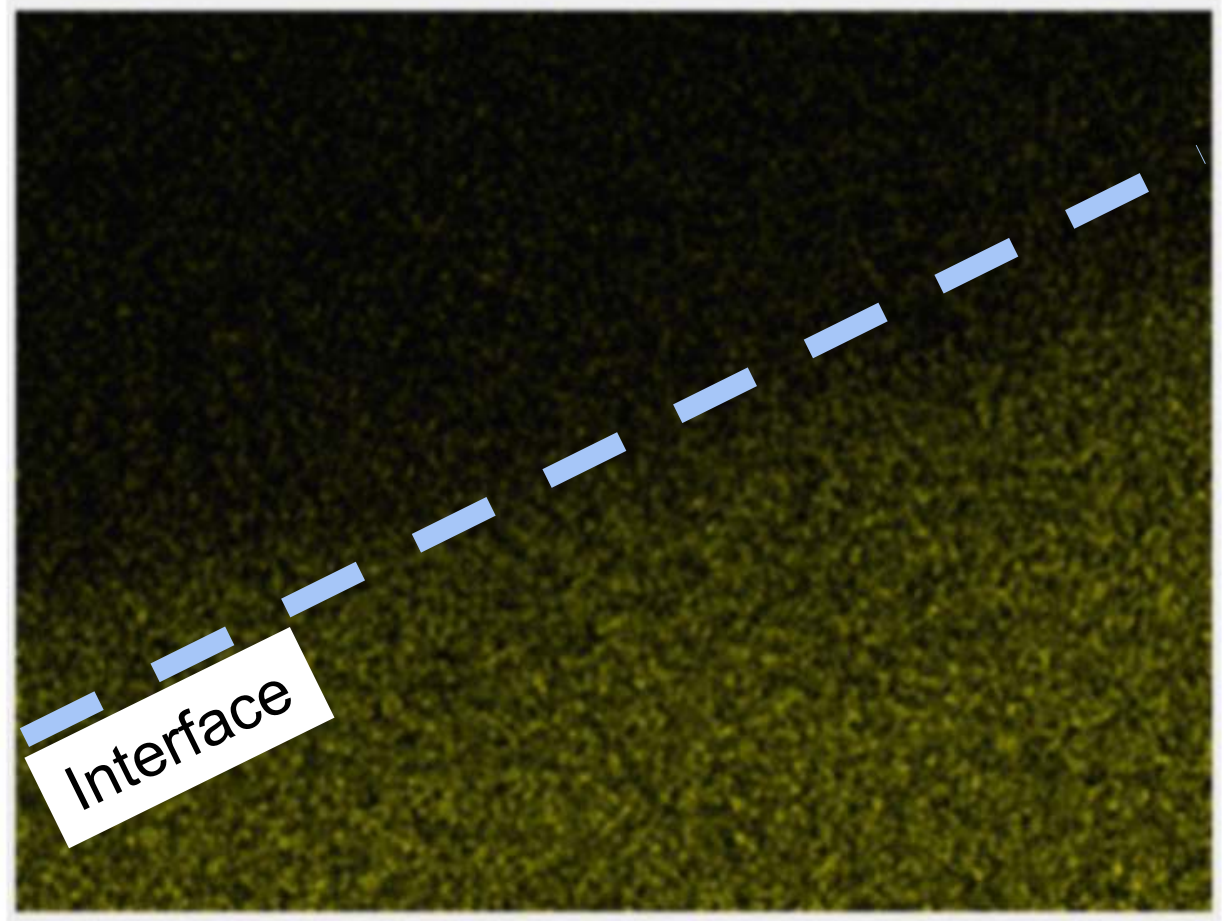

$100 \mu \mathrm{m}$

- $\mathrm{F}$ and $\mathrm{S}$ atoms diffuse across interface 


\section{2) Interdiffusion at Interface}

After Cure

EDS

Line

Scan

Data

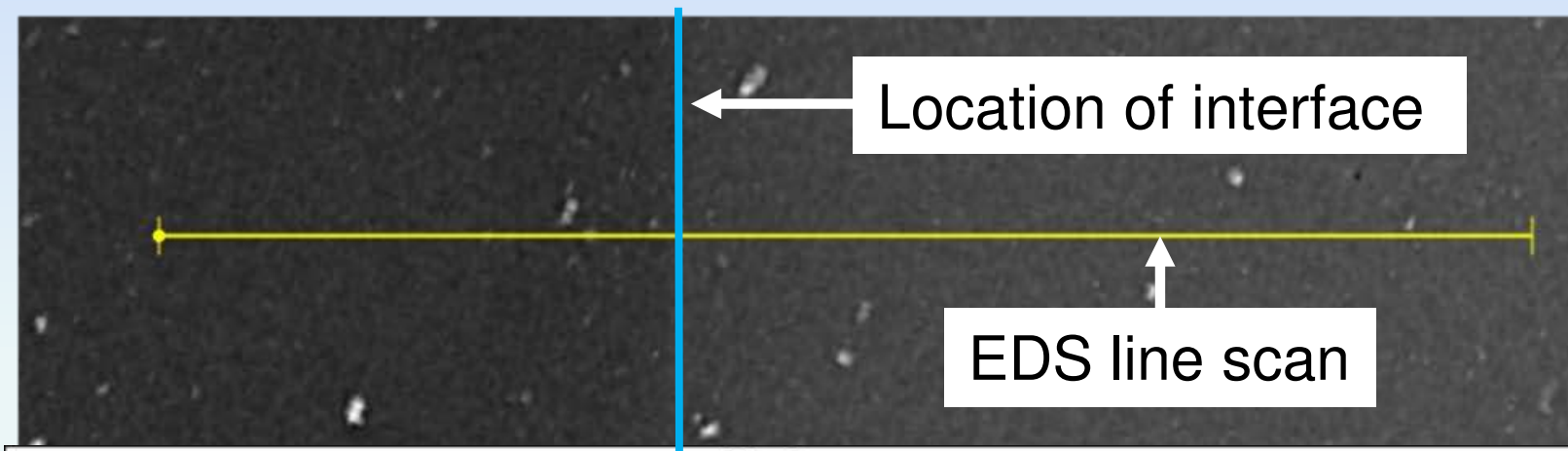

$\mathrm{F}$ atomic concentration

Before Cure

S atomic concentration Before Cure

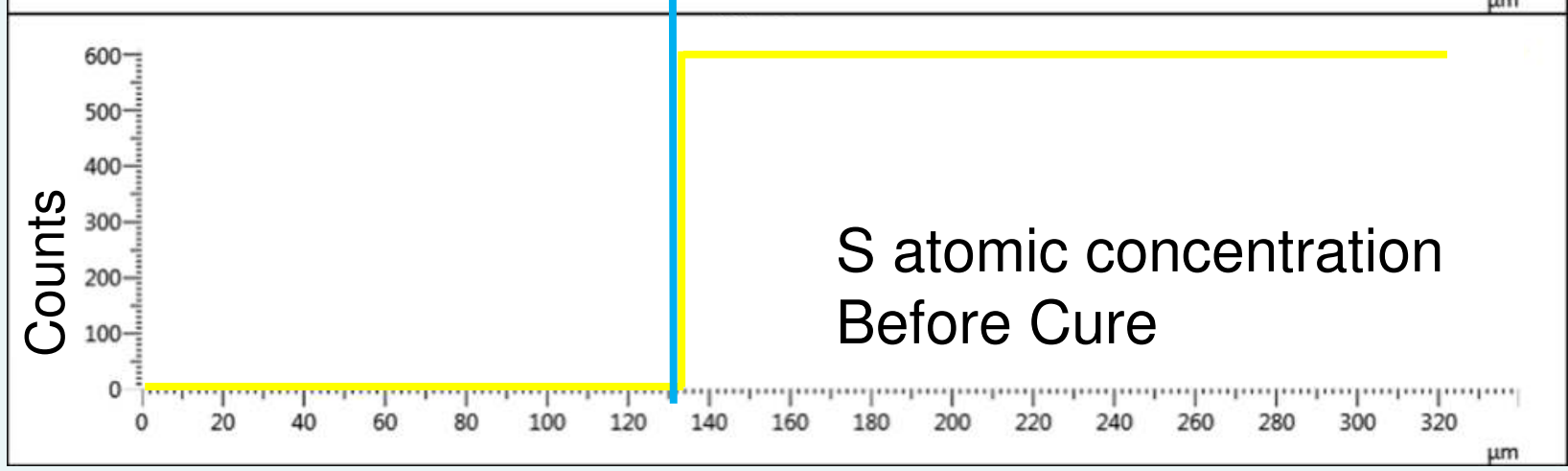




\section{Variation in Curved Beam Test Data}

- X-Ray Computed Tomography Inspection

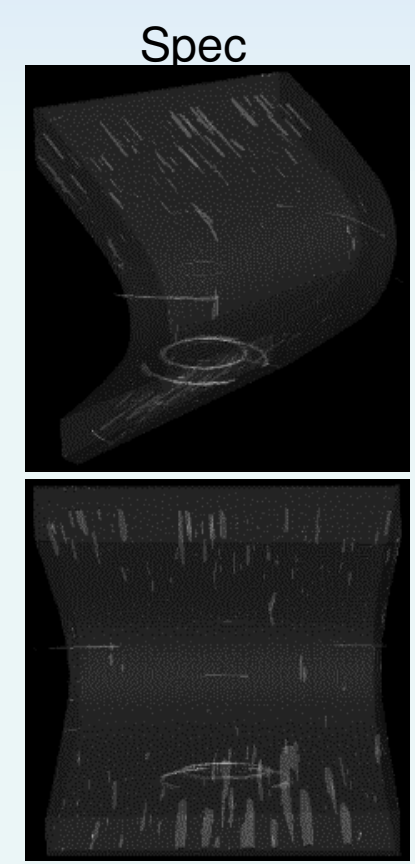

Low porosity

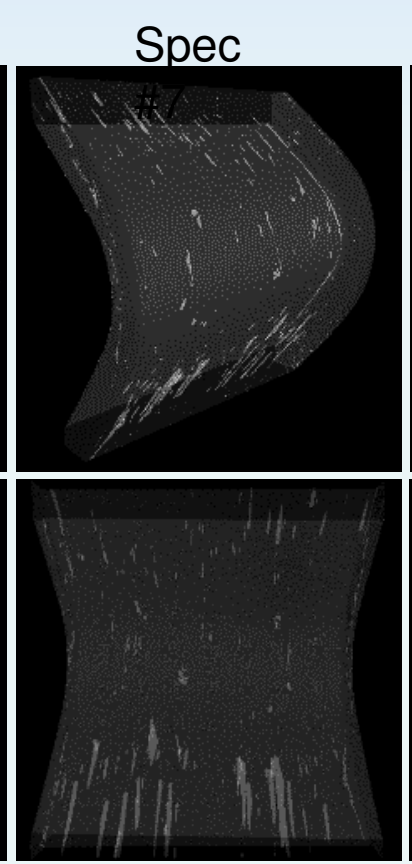

Low porosity

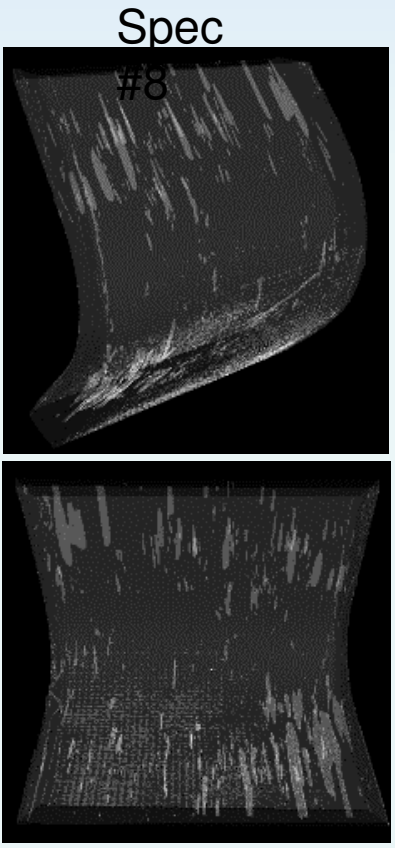

High porosity

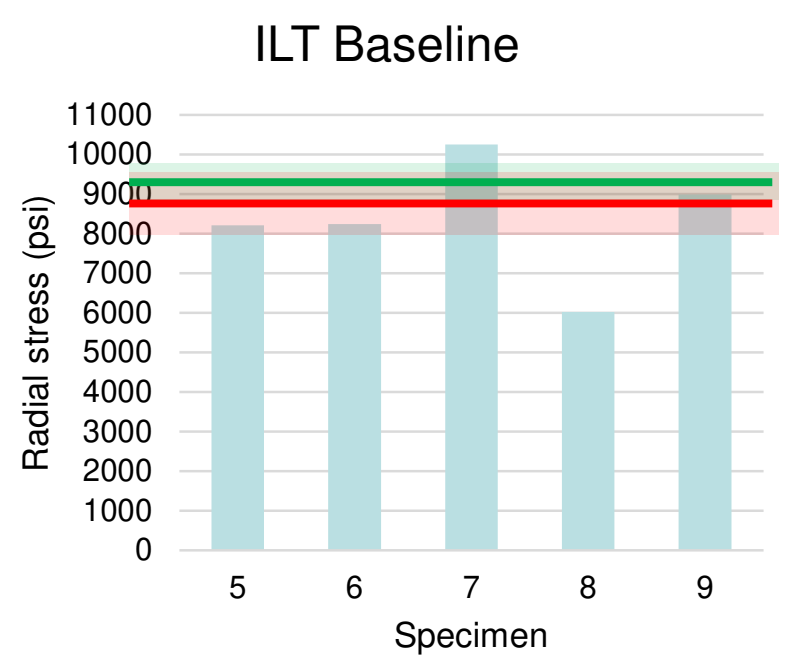

Ave Str: 8341 psi (w/out 8): 8921 psi Std Dev: 1377 psi (w/out 8): 830 psi Cof Var: $\quad 17 \%$ (w/out 8): $\quad 9 \%$ Low porosity

\begin{tabular}{|c|c|c|c|}
\hline 1 & Goals for: & DOE $1(50 \%)$ & DOE $2(80 \%)$ \\
\hline$(w / 8)$ & ILS (psi) & 4171 & 6673 \\
\hline (w/out 8) & ILS (psi) & 4461 & 7137 \\
\hline
\end{tabular}




\section{Double Cantilever Beam (DCB) Test}

Mode-I Interlaminar Fracture Toughness

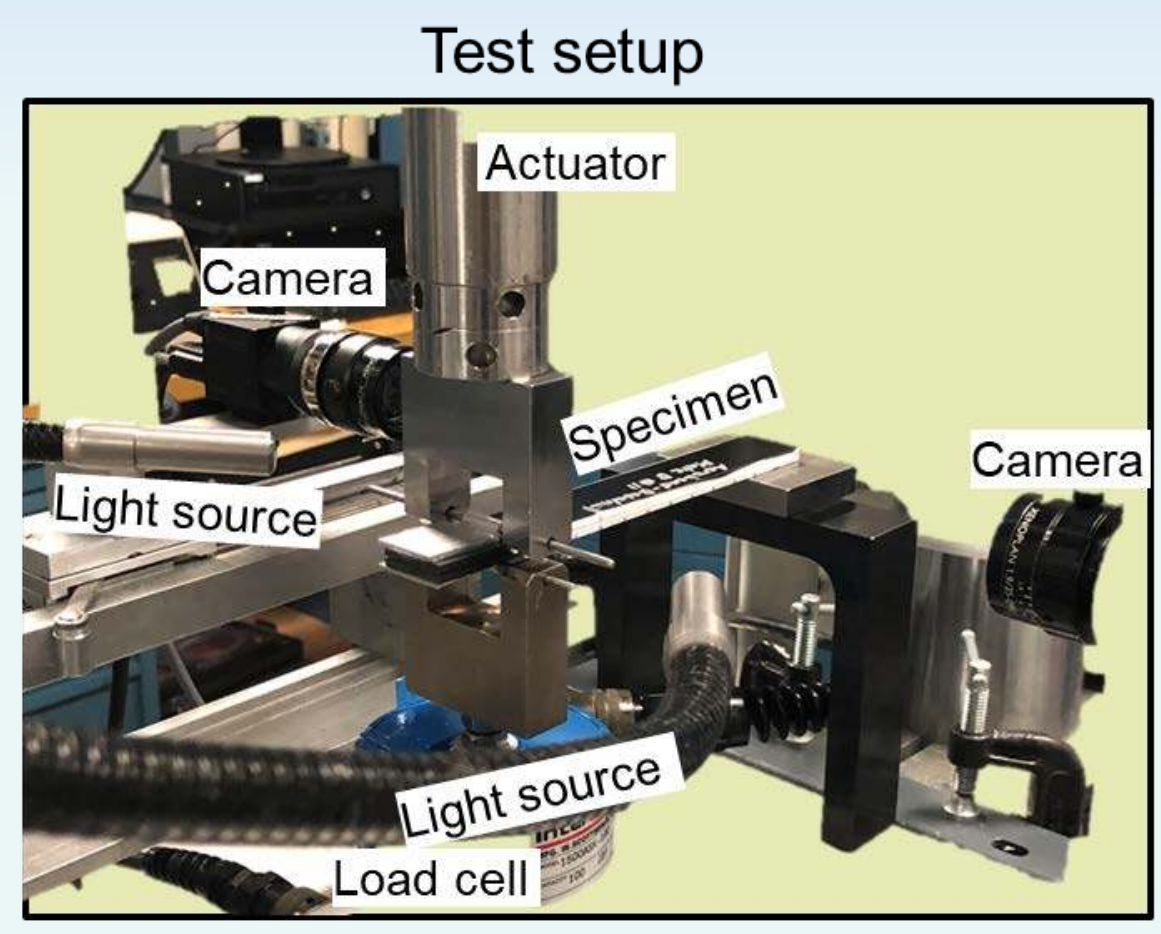

\begin{tabular}{|l|c|c|}
\hline $\mathbf{G}_{\mathbf{I c}}\left(\mathbf{J} / \mathbf{m}^{2}\right)$ & Round 1 (50\%) & Round 2 (80\%) \\
\hline Goals & 102 & 161 \\
\hline Measured & $39 \pm 16(19 \%)$ & -- \\
\hline
\end{tabular}

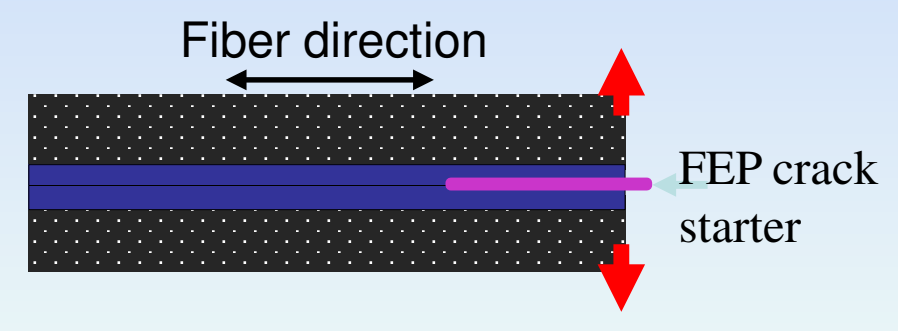

Short of goal. AERoBOND resins are not toughened like commercial systems

Baseline Properties

Ave $\mathrm{G}_{\mathrm{Ic}-\mathrm{ss}}: 203 \mathrm{~J} / \mathrm{m}^{2}$

Std Dev: $8.94 \mathrm{~J} / \mathrm{m}^{2}$

Cof Var: $\quad 4.4 \%$

Statistics for $\Delta a>0.6$ in. (steady state) 


\section{Single-Lap Shear (SLS) Test}

\section{$\underline{\text { Test Specimen }}$}

\section{Pre-test}

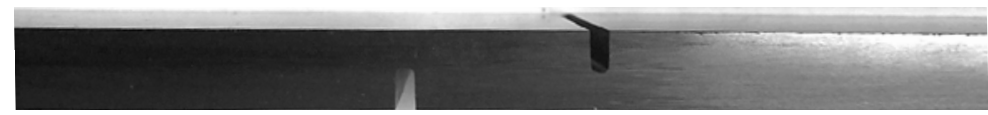

\section{Post-test}
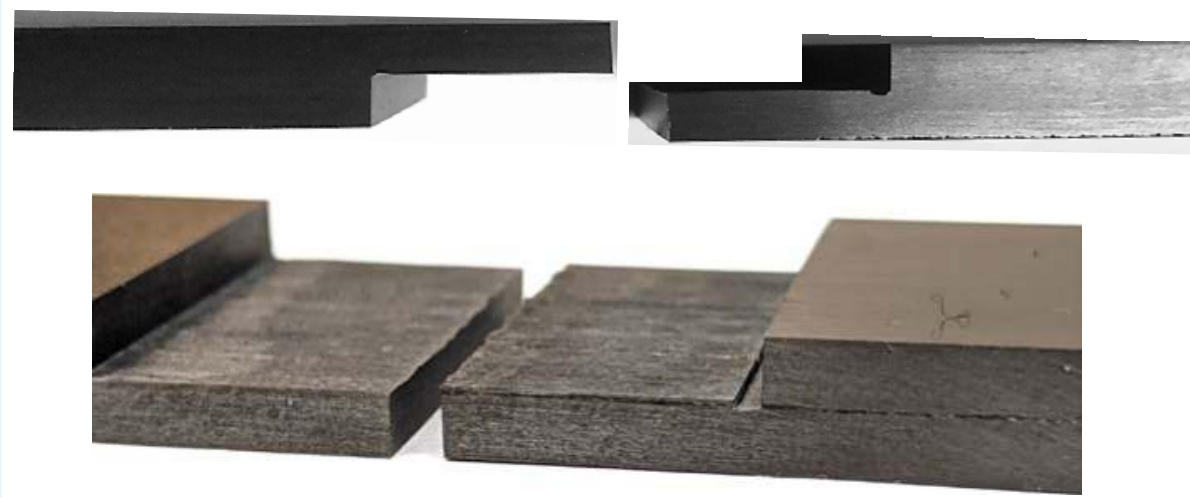

\begin{tabular}{|l|c|c|}
\hline ILS (MPa) & Round 1 (50\%) & Round 2 (80\%) \\
\hline Goals & 15.7 & 25.1 \\
\hline Measured & $25.7 \pm 2.2(82 \%)$ & -- \\
\hline
\end{tabular}

Interlaminar Shear Strength

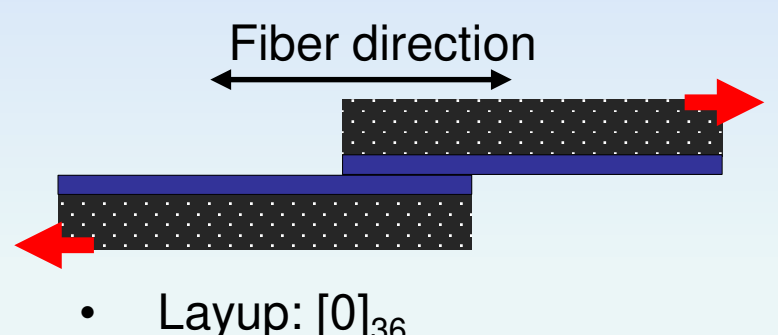

Specimens often failed away from AERoBOND interface due to stress concentrations at different depths in cross-section.

Baseline Properties

Ave ILS: $\mathbf{3 1 . 4} \mathrm{MPa}$

Std Dev: $1.6 \mathrm{MPa}$

Cof Var: $4.35 \%$ 


\section{Double-Lap Shear (DLS) Test}

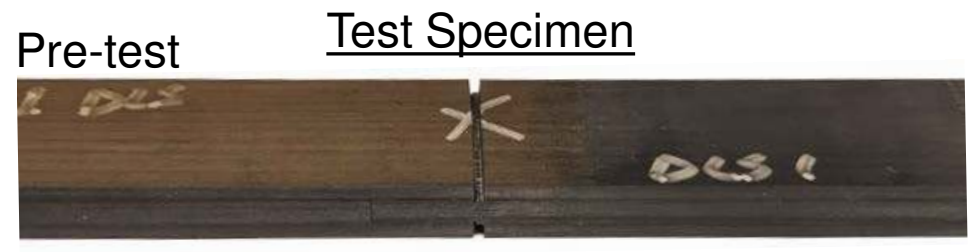

\section{Post-test}

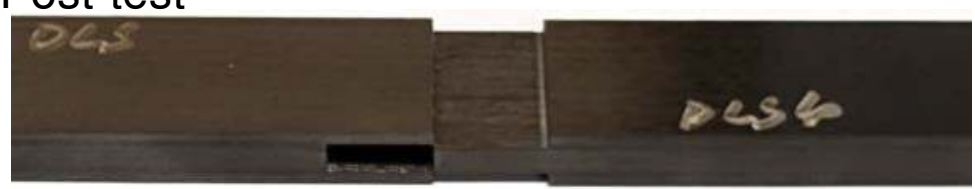

Interlaminar Shear Strength

\section{Post-test}

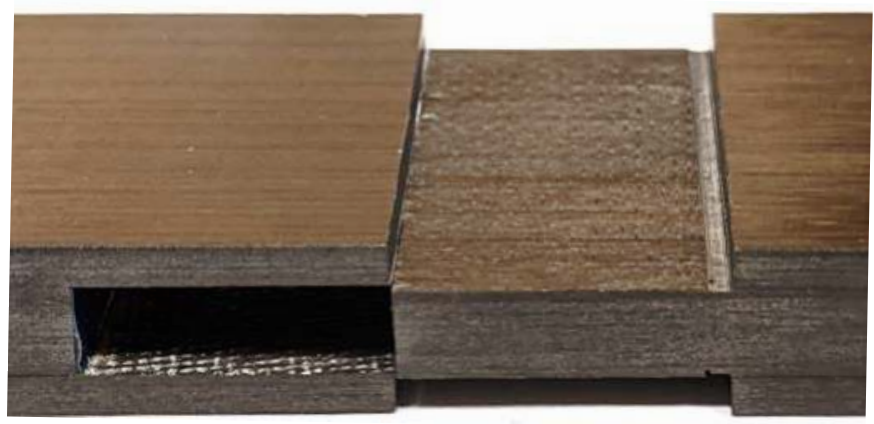

Lower than expected properties that may be related to complexity of fabrication.

\begin{tabular}{|l|c|c|}
\hline ILS (MPa) & Round 1 (50\%) & Round 2 (80\%) \\
\hline Goals & 20.8 & 33.3 \\
\hline Measured & $9.8 \pm 1.5(24 \%)$ & --
\end{tabular}

Baseline Properties Ave ILS: $41.6 \mathrm{MPa}$ Std Dev: $2.2 \mathrm{MPa}$ Cof Var: $5.17 \%$ 


\section{Curved Beam (CB) Test}

Interlaminar Tensile Strength

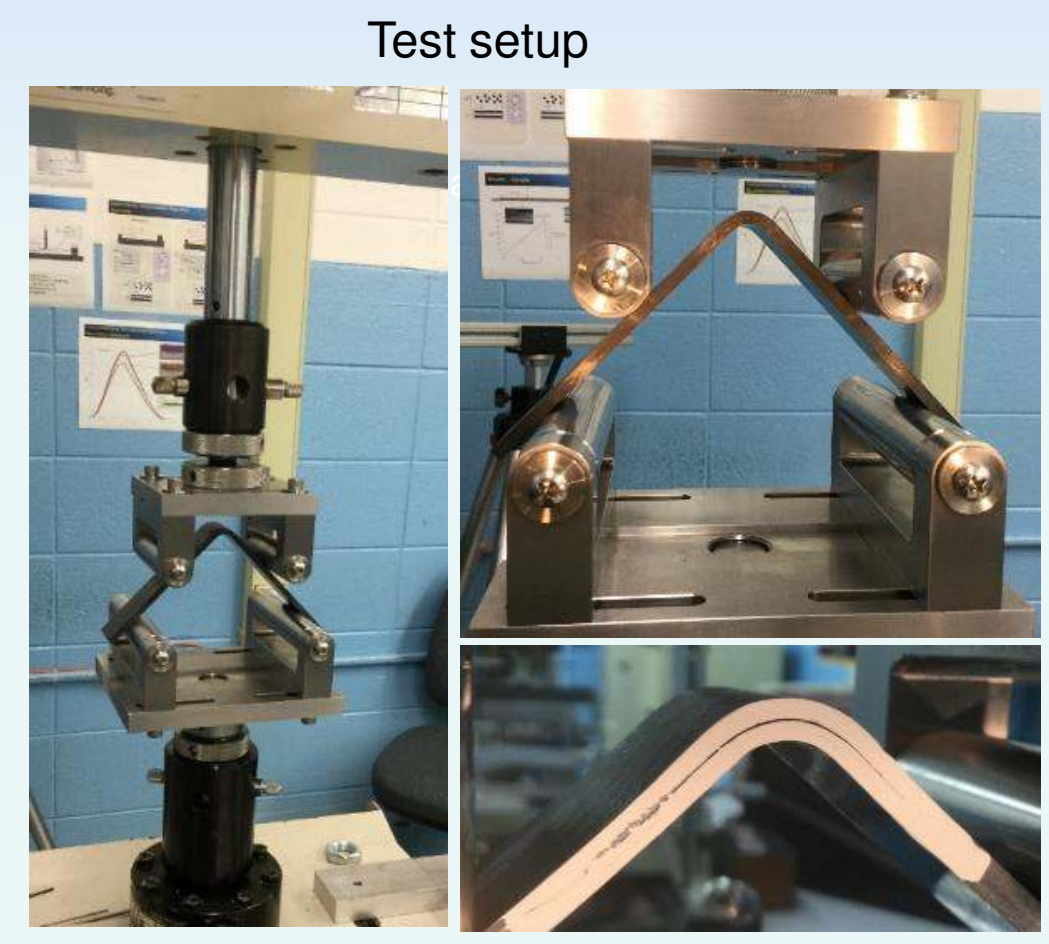

\begin{tabular}{|l|c|c|}
\hline ILT (MPa) & Round 1 (50\%) & Round 2 (80\%) \\
\hline Goals & 28.8 & 46.0 \\
\hline
\end{tabular}

Properties are near $50 \%$ goal and surprisingly good based on complexity of build. No matched tooling was available to make matched "L" shaped parts.

Baseline Properties Ave ILT: $57.5 \mathrm{MPa}$

Std Dev: $9.5 \mathrm{MPa}$

Cof Var: $17 \%$ 


\section{Flatwise Tension (FWT) Test}
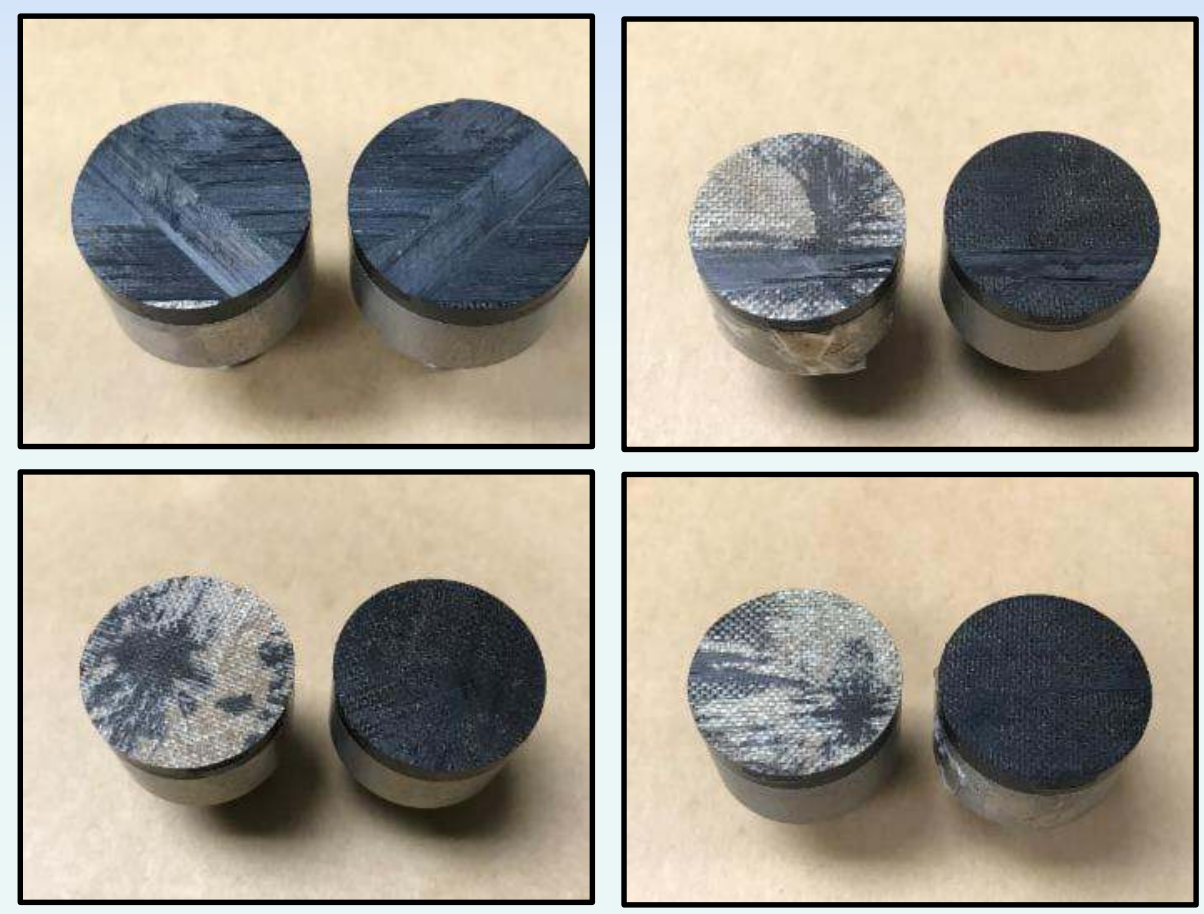

Interlaminar Tensile Strength

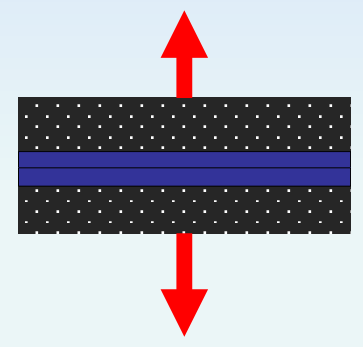

Panels failed at the AERoBOND joint near the ER-to-HR interface in most cases. Failure locus is away from machined surface indicating an accurate measurement.

\begin{tabular}{|l|c|c|c|}
\hline ILT (MPa) & Round 1 (50\%) & Round 2 (80\%) & Baseline Properties \\
\hline Goals & 32.1 & 33.4 & Ave ILT: $64.1 \mathrm{MPa}$ \\
\hline Measured & $37.5(59 \%)$ & -- & Std Dev: $6.4 \mathrm{MPa}$ \\
\hline
\end{tabular}




\section{Preliminary Impact Testing}

\section{Pre-Impact C-Scan Image}

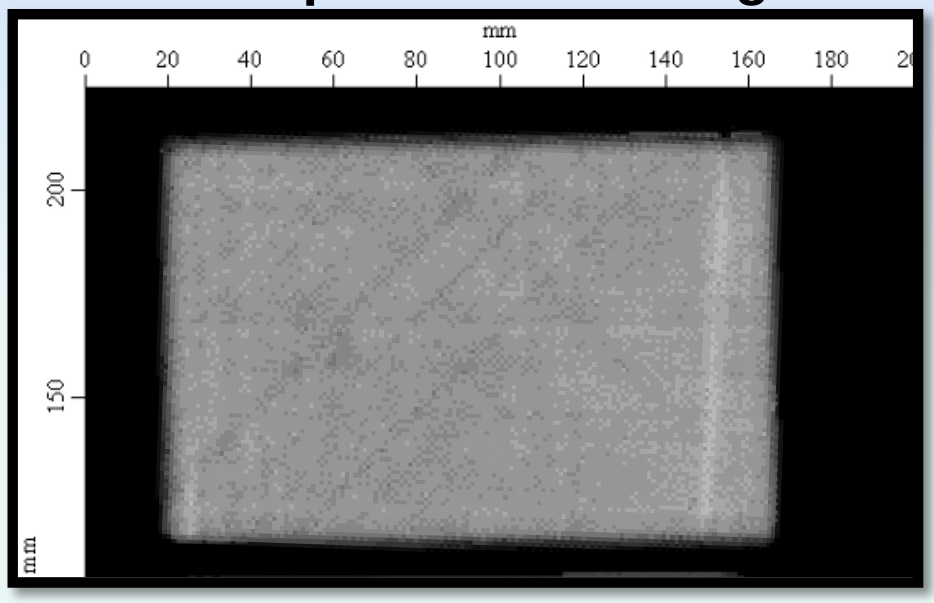

Step 1
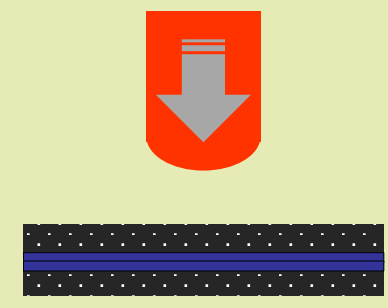

Post-Impact Inspection

(Impact energy: $5.5 \mathrm{~J}$ )
Damage too close to clamped region at edge of panel. Re-evaluating impact energy.


Step 2

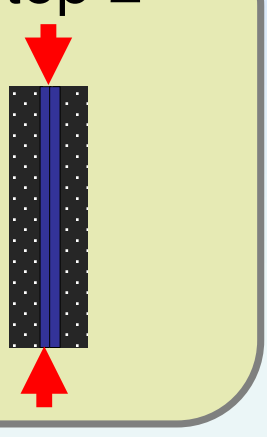

